Communications in Mathematical Physics, 184, no. 1 (1997), 95-117

DOI: $10.1007 / \mathrm{s} 002200050054$

The original publication is available at www.springerlink.com/content/9c34gj7gm38xglta/

\title{
GEOMETRICAL MEANING OF $R$-MATRIX ACTION FOR QUANTUM GROUPS AT ROOTS OF 1
}

\author{
FABio Gavarini \\ Dipartimento di Matematica, Istituto G. Castelnuovo, \\ Università degli studi di Roma "La Sapienza"
}

\begin{abstract}
The present work splits in two parts: first, we perform a straightforward generalization of results from [Re], proving that quantum groups $U_{q}^{M}(\mathfrak{g})$ and their unrestricted specializations at roots of 1 , in particular the function algebra $F[H]$ of the Poisson group $H$ dual of $G$, are braided; second, as a main contribution, we prove the convergence of the (specialized) $R$-matrix action to a birational automorphism of a $2 \ell$-fold ramified covering of $\operatorname{Spec}\left(U_{\varepsilon}^{M}(\mathfrak{g})\right)^{\times 2}$ when $\varepsilon$ is a primitive $\ell$-th root of 1 , and of a 2-fold ramified covering of $H$, thus giving a geometric content to the notion of triangularity (or braiding) for quantum groups at roots of 1 .
\end{abstract}

\section{$\S 1$ Definitions}

1.1 Cartan data. Let $\mathfrak{g}$ be a complex finite dimensional semisimple Lie algebra of rank $n$, with Cartan matrix $A:=\left(a_{i j}\right)_{i, j=1, \ldots, n}$; let $R$ be its root system, $Q$, resp. $P$, be its root lattice, resp. weight lattice; we fix a subset $R^{+}(\subset R)$ of positive roots, a basis $\left\{\alpha_{1}, \ldots, \alpha_{n}\right\}\left(\subset R^{+}\right)$of simple roots, and we let $\left\{\omega_{1}, \ldots, \omega_{n}\right\}$ be the dual basis of $P$. We denote by $W$ the Weyl group of $\mathfrak{g}$, with generators $s_{1}, \ldots, s_{n}$ (namely the reflections associated with simple roots), and we set $N:=\#\left(R^{+}\right)$. Finally, we let $\left(d_{1}, \ldots, d_{n}\right)$ be the (unique) $n$-tuple of relatively prime positive integers such that $\left(d_{i} a_{i j}\right)_{i, j=1, \ldots, n}$ is a symmetric positive definite matrix.

1.1 Quantum enveloping algebras. We briefly recall some definitions. The quantized universal enveloping algebra $U_{h}(\mathfrak{g})$ is the associative algebra with 1 over $\mathbb{C}[[h]]$ generated by $Y_{1}, \ldots, Y_{n}, H_{1}, \ldots, H_{n}, X_{1}, \ldots, X_{n}$ with relations (for $i, j=1, \ldots, n$ )

$$
\begin{gathered}
H_{i} H_{j}-H_{j} H_{i}=0 \\
H_{i} X_{j}-X_{j} H_{i}=a_{i j} X_{j}, \quad H_{i} Y_{j}-Y_{j} H_{i}=-a_{i j} Y_{j} \\
X_{i} Y_{j}-Y_{j} X_{i}=\delta_{i j} \frac{\exp \left(h d_{i} H_{i}\right)-\exp \left(-h d_{i} H_{i}\right)}{\exp \left(h d_{i}\right)-\exp \left(-h d_{i}\right)}
\end{gathered}
$$




$$
\begin{array}{ll}
\sum_{k=0}^{1-a_{i j}}(-1)^{k}\left[\begin{array}{c}
1-a_{i j} \\
k
\end{array}\right]_{q_{i}} X_{i}^{1-a_{i j}-k} X_{j} X_{i}^{k}=0 & \forall i \neq j \\
\sum_{k=0}^{1-a_{i j}}(-1)^{k}\left[\begin{array}{c}
1-a_{i j} \\
k
\end{array}\right]_{q_{i}} Y_{i}^{1-a_{i j}-k} Y_{j} Y_{i}^{k}=0 & \forall i \neq j
\end{array}
$$

where $q:=\exp (h), q_{i}:=q^{d_{i}}=\exp \left(h d_{i}\right)$, and the Gaussian binomial $\left[\begin{array}{c}m \\ n\end{array}\right]_{q}$ is defined by

$$
\left[\begin{array}{c}
m \\
n
\end{array}\right]_{q}:=\frac{[m]_{q} !}{[m-n]_{q} ![n]_{q} !}, \quad[k]_{q} !:=\prod_{s=1}^{k}[s]_{q}, \quad[s]_{q}:=\frac{q^{s}-q^{-s}}{q-q^{-1}}
$$

for all $m, n, k, s \in \mathbb{N}_{+}, n \leq m$, with $[s]_{q},[k]_{q} !,\left[\begin{array}{c}m \\ n\end{array}\right]_{q} \in \mathbb{C}\left[q, q^{-1}\right]$. It is known that $U_{h}(\mathfrak{g})$ has a Hopf algebra structure, given by $(i=1, \ldots, n)$

$$
\begin{array}{cccc}
\Delta\left(Y_{i}\right):=Y_{i} \otimes \exp \left(-h d_{i} H_{i}\right)+1 \otimes Y_{i}, & S\left(Y_{i}\right):=-Y_{i} \exp \left(h d_{i} H_{i}\right), & \epsilon\left(Y_{i}\right):=0 \\
\Delta\left(H_{i}\right):=H_{i} \otimes 1+1 \otimes H_{i}, & S\left(H_{i}\right):=-H_{i}, & \epsilon\left(H_{i}\right):=0 \\
\Delta\left(X_{i}\right):=X_{i} \otimes 1+\exp \left(h d_{i} H_{i}\right) \otimes X_{i}, & S\left(X_{i}\right):=-\exp \left(-h d_{i} H_{i}\right) X_{i}, & \epsilon\left(X_{i}\right):=0 .
\end{array}
$$

Let $M$ be a lattice such that $Q \leq M \leq P$ : the quantized universal enveloping algebra $U_{q}^{M}(\mathfrak{g})$ (cf. $\left.[\mathrm{DP}], \S 9\right)$ is the associative algebra with 1 over $\mathbb{C}(q)$ generated by $F_{1}, \ldots, F_{n}$, $L_{\mu}(\forall \mu \in M), E_{1}, \ldots, E_{n}$ with relations $(i, j=1, \ldots, n ; \mu, \nu \in M)$

$$
\begin{gathered}
L_{\mu} L_{\nu}=L_{\mu+\nu}=L_{\nu} L_{\mu} \quad, \quad L_{0}=1 \\
L_{\mu} E_{j}=q^{\left\langle\mu \mid \alpha_{j}\right\rangle} E_{j} L_{\mu}, \quad L_{\mu} F_{j}=q^{-\left\langle\mu \mid \alpha_{j}\right\rangle} F_{j} L_{\mu} \\
E_{i} F_{j}-F_{j} E_{i}=\delta_{i j} \frac{L_{\alpha_{i}}-L_{-\alpha_{i}}}{q_{i}-q_{i}^{-1}} \\
\sum_{k=0}^{1-a_{i j}}(-1)^{k}\left[\begin{array}{c}
1-a_{i j} \\
k
\end{array}\right]_{q_{i}} E_{i}^{1-a_{i j}-k} E_{j} E_{i}^{k}=0 \\
\sum_{k=0}^{1-a_{i j}}(-1)^{k}\left[\begin{array}{c}
1-a_{i j} \\
k
\end{array}\right]_{q_{i}} F_{i}^{1-a_{i j}-k} F_{j} F_{i}^{k}=0
\end{gathered}
$$

A Hopf algebra structure on $U_{q}^{M}(\mathfrak{g})$ is defined by $(i=1, \ldots, n ; \mu \in M)$

$$
\begin{array}{cccc}
\Delta\left(F_{i}\right):=F_{i} \otimes L_{-\alpha_{i}}+1 \otimes F_{i}, & S\left(F_{i}\right):=-F_{i} L_{\alpha_{i}}, & \epsilon\left(F_{i}\right):=0 \\
\Delta\left(L_{\mu}\right):=L_{\mu} \otimes L_{\mu}, & S\left(L_{\mu}\right):=L_{-\mu}, & \epsilon\left(L_{\mu}\right):=1 \\
\Delta\left(E_{i}\right):=E_{i} \otimes 1+L_{\alpha_{i}} \otimes E_{i}, & S\left(E_{i}\right):=-L_{-\alpha_{i}} E_{i}, & \epsilon\left(E_{i}\right):=0 .
\end{array}
$$

It is clear that $U_{q}^{M}(\mathfrak{g}) \hookrightarrow U_{q}^{M^{\prime}}(\mathfrak{g})$ whenever $Q \leq M \leq M^{\prime} \leq P$, this being a Hopf algebra embedding. In the sequel we shall also use notation $L_{i}:=L_{\omega_{i}}, K_{i}:=L_{\alpha_{i}}$ (for all $i=1, \ldots, n)$. 
The very definitions imply the existence of a Hopf algebra monomorphism

$$
j: U_{q}^{Q}(\mathfrak{g}) \longleftrightarrow U_{h}(\mathfrak{g})
$$

given by $q \mapsto \exp (h), F_{i} \mapsto Y_{i}, K_{i} \mapsto \exp \left(h d_{i} H_{i}\right), E_{i} \mapsto X_{i}$; still from definitions it is also clear that this uniquely extends to an embedding

$$
j: U_{q}^{M}(\mathfrak{g}) \longleftrightarrow U_{h}(\mathfrak{g})
$$

for all lattices $M$; in particular $U_{q}^{P}(\mathfrak{g}) \longleftrightarrow U_{h}(\mathfrak{g})$. Finally, we shall denote by $U_{q}^{M}\left(\mathfrak{b}_{+}\right)$, resp. $U_{q}^{M}\left(\mathfrak{b}_{-}\right)$, the Hopf subalgebra of $U_{q}^{M}(\mathfrak{g})$ generated by $L_{\mu}$ 's and $E_{i}$ 's, resp. $F_{i}$ 's: these are called quantum Borel subalgebras.

An interesting property that Hopf algebras can enjoy is quasitriangularity:

Definition 1.2. (cf. [Dr]) A Hopf algebra $H$ is called quasitriangular if there exists an invertible element $R \in H \otimes H$ (or an element of an appropriate completion of $H \otimes H$ ) such that

$$
\begin{gathered}
R \cdot \Delta(a) \cdot R^{-1}=\operatorname{Ad}(R)(\Delta(a))=\Delta^{\mathrm{op}}(a) \\
(\Delta \otimes \mathrm{id})(R)=R_{13} R_{23} \\
(\mathrm{id} \otimes \Delta)(R)=R_{13} R_{12}
\end{gathered}
$$

where $\Delta^{\mathrm{op}}$ is the opposite comultiplication, i. e. $\Delta^{\mathrm{op}}(a)=\sigma \circ \Delta(a)$ with $\sigma: A^{\otimes 2} \rightarrow A^{\otimes 2}$, $a \otimes b \mapsto b \otimes a$, and $R_{12}, R_{13}, R_{23} \in H^{\otimes 3} \quad$ (or the appropriate completion of $H^{\otimes 3}$ ), $R_{12}=$ $R \otimes 1, R_{23}=1 \otimes R, R_{13}=(\sigma \otimes \mathrm{id})\left(R_{23}\right)=(\mathrm{id} \otimes \sigma)\left(R_{12}\right)$.

As a corollary of this definition, $R$ satisfies the Yang-Baxter equation in $H^{\otimes 3}$ (cf. [Ta])

$$
R_{12} R_{13} R_{23}=R_{23} R_{13} R_{12}
$$

so that a braid group action is defined on tensor products of $H$-modules. The quantum universal enveloping algebra $U_{h}(\mathfrak{g})$ is quasitriangular (cf. [Dr], [LS], [KR]): its $R$-matrix is

$$
R=\prod_{\alpha \in R^{+}} \exp _{q_{\alpha}}\left(\left(q_{\alpha}{ }^{-1}-q_{\alpha}\right) X_{\alpha} \otimes Y_{\alpha}\right) \cdot \exp \left(-h \sum_{i, j=1}^{n} B_{i j} H_{i} \otimes H_{j}\right)
$$

where $\prod_{\alpha \in R^{+}}$denotes an ordered product (with respect to a fixed convex ordering of $R^{+}$), $q_{\alpha}:=q^{d_{\alpha}}$ (where $d_{\alpha}$ is one-half the square length of the root $\alpha$; in particular $d_{\alpha_{i}}=d_{i}$ for all $i),\left(B_{i j}\right)_{i, j=1, \ldots, n}:=\left(d_{i} a_{i j}\right)_{i, j=1, \ldots, n}^{-1}$, and $X_{\alpha}, Y_{\alpha}$ are $q$-analogue of root vectors (not unique, however) attached to roots $\alpha,-\alpha$. On the other hand, this is not true - strictly speaking — for the $\mathbb{C}(q)$-algebras $U_{q}^{M}(\mathfrak{g})$ : to be precise we need a slight modification of the notion of quasitriangularity, suggested by Reshetikin, as follows: 
Definition 1.3. (cf. [Re], Definition 2) A Hopf algebra $H$ is called braided if there exists an automorphism $\mathcal{R}$ of $H \otimes H$ (or of an appropriate completion of $H \otimes H$ ) distinct from $\sigma: a \otimes b \mapsto b \otimes a$ such that

$$
\begin{gathered}
\mathcal{R} \circ \Delta=\Delta^{\text {op }} \\
(\Delta \otimes \mathrm{id}) \circ \mathcal{R}=\mathcal{R}_{13} \circ \mathcal{R}_{23} \circ(\Delta \otimes \mathrm{id}) \\
(\mathrm{id} \otimes \Delta) \circ \mathcal{R}=\mathcal{R}_{13} \circ \mathcal{R}_{12} \circ(\mathrm{id} \otimes \Delta)
\end{gathered}
$$

Here $\mathcal{R}_{12}, \mathcal{R}_{13}, \mathcal{R}_{23}$ are the automorphisms of $H \otimes H \otimes H$ defined by $\mathcal{R}_{12}=\mathcal{R} \otimes \mathrm{id}$, $\mathcal{R}_{23}=\mathrm{id} \otimes \mathcal{R}, \mathcal{R}_{13}=(\sigma \otimes \mathrm{id}) \circ(\mathrm{id} \otimes \mathcal{R}) \circ(\sigma \otimes \mathrm{id})$.

It follows from this definition that $\mathcal{R}$ satisfies the Yang-Baxter equation in $\operatorname{End}\left(H^{\otimes 3}\right)$ :

$$
\mathcal{R}_{12} \circ \mathcal{R}_{13} \circ \mathcal{R}_{23}=\mathcal{R}_{23} \circ \mathcal{R}_{13} \circ \mathcal{R}_{12}
$$

which yields a braid group action on tensor powers of $H$. Furthermore, it is clear that if $(H, R)$ is quasitriangular, then $(H, \operatorname{Ad}(R))$ is braided. Again from [Re] we resume another definition (slightly modified indeed).

Definition 1.4. (cf. [Re], Definition 3) Let $H$ be a Hopf algebra, let $\mathcal{R}^{(0)}$ be an algebra automorphism of $H \otimes H$ and $R^{(1)} \in H \otimes H$ an invertible element such that

$$
\begin{gathered}
R^{(1)} \cdot \mathcal{R}^{(0)}(\Delta(a)) \cdot R^{(1)^{-1}}=\left(\operatorname{Ad}\left(R^{(1)}\right) \circ \mathcal{R}^{(0)}\right)(\Delta(a))=\Delta^{\mathrm{op}}(a) \\
(\Delta \otimes \mathrm{id}) \circ \mathcal{R}^{(0)}=\mathcal{R}^{(0)}{ }_{13} \circ \mathcal{R}^{(0)}{ }_{23} \circ(\Delta \otimes \mathrm{id}) \\
(\mathrm{id} \otimes \Delta) \circ \mathcal{R}^{(0)}=\mathcal{R}^{(0)}{ }_{13} \circ \mathcal{R}^{(0)}{ }_{12} \circ(\mathrm{id} \otimes \Delta) \\
(\Delta \otimes \mathrm{id})\left(R^{(1)}\right)=R^{(1)}{ }_{13} \cdot \mathcal{R}^{(0)}{ }_{13}\left(R^{(1)}{ }_{23}\right) \\
(\mathrm{id} \otimes \Delta)\left(R^{(1)}\right)=R^{(1)}{ }_{13} \cdot \mathcal{R}^{(0)}{ }_{13}\left(R_{12}^{(1)}\right)
\end{gathered}
$$

then $\left(H, \operatorname{Ad}\left(R^{(1)}\right) \circ \mathcal{R}^{(0)}\right)$ is a braided Hopf algebra, and the element $R^{(1)}$ is called the universal $R$-matrix of $\left(H, \operatorname{Ad}\left(R^{(1)}\right) \circ \mathcal{R}^{(0)}\right)$.

Finally we recall from $[\mathrm{Ta}]$ the strictly related notion below:

Definition 1.5. (cf. [Ta], $\S 4)$ Let $H$ be a Hopf algebra, let $\Phi$ be an algebra automorphism of $H \otimes H$ and $C \in H \otimes H$ be an invertible element such that

$$
\begin{gathered}
C^{-1} \cdot \Phi\left(\Delta^{\mathrm{op}}(a)\right) \cdot C=\Delta(a) \\
\left(\Phi_{23} \circ \Phi_{13}\right)\left(C_{12}\right)=C_{12} \\
\left(\Phi_{12} \circ \Phi_{13}\right)\left(C_{23}\right)=C_{23} \\
(\Delta \otimes \mathrm{id})(C)=\Phi_{23}\left(C_{13}\right) \cdot C_{23} \\
(\mathrm{id} \otimes \Delta)(C)=\Phi_{12}\left(C_{13}\right) \cdot C_{12}
\end{gathered}
$$

then we will say that $(H, C, \Phi)$ is a pretriangular Hopf algebra. 


\section{$\S 2$ Some $q$-calculus}

2.1. In this section we introduce some material to be used in the sequel; as standard references for $q$-special functions and related matters we quote [Ex] and [GR].

Let us introduce some $q$-symbols. We have $q$-numbers

$$
(s)_{q}:=\frac{q^{s}-1}{q-1}, \quad(k)_{q} !:=\prod_{s=1}^{k}(s)_{q}, \quad\left(\begin{array}{c}
m \\
n
\end{array}\right)_{q}:=\frac{(m)_{q} !}{(m-n)_{q} !(n)_{q} !}
$$

for $s, k, m, n \in \mathbb{N}_{+}, n \leq m$, with $(s)_{q},(k)_{q} !,\left(\begin{array}{c}m \\ n\end{array}\right)_{q} \in \mathbb{C}\left[q, q^{-1}\right]$, and the symbol $(a ; q)_{n}:=$ $\prod_{k=0}^{n-1}\left(1-a q^{k}\right)$, for $n \in \mathbb{N}, a \in \mathbb{C}$. Now consider the function of $z$

$$
(z ; q)_{\infty}:=\prod_{n=0}^{\infty}\left(1-z q^{n}\right)
$$

we regard it as an element of $\mathbb{C}(q)[[z]]$. The infinite product expressing $(z ; q)_{\infty}$ converges to an analytic function of $z$ in any finite part of $\mathbb{C}$ if $q$ is a complex number such that $|q|<1$; its Taylor series is then

$$
(z ; q)_{\infty}=\sum_{n=0}^{\infty} \frac{(-1)^{n} q^{\left(\begin{array}{c}
n \\
2
\end{array}\right)}}{(q ; q)_{n}} z^{n}
$$

Also the series

$$
e_{q}(z):=\sum_{n=0}^{\infty} \frac{1}{(q ; q)_{n}} z^{n}, \quad E_{q}(z):=\sum_{n=0}^{\infty} \frac{q^{\left(\begin{array}{c}
n \\
2
\end{array}\right)}}{(q ; q)_{n}} z^{n}
$$

both converge to analytic functions of $z$; moreover, one has

$$
e_{q}(z)=(z ; q)_{\infty}^{-1}, \quad E_{q}(z)=(-z ; q)_{\infty}
$$

so that $E_{q}(-z) e_{q}(z)=1$. Finally

$$
\exp _{q}(z):=\sum_{n=0}^{\infty} \frac{1}{(n)_{q^{2}} !} z^{n}
$$

thus one has

$$
\exp _{q}(z)=e_{q^{2}}\left(\left(1-q^{2}\right) z\right)
$$

We claimed above that $(z ; q)_{\infty}$ is an analytic function of $z$ for $|q|<1$; the following lemma describes the behavior of this function for $q \rightarrow \varepsilon$, $\varepsilon$ a root of 1 . 
Lemma 2.2. ([Re], Lemma 3.4.1) Let $\varepsilon$ be a primitive $\ell$-th root of 1 , with $\ell$ odd. The asymptotic behavior of the function $(o f q)(z ; q)_{\infty}$ for $q \rightarrow \varepsilon$ is given by

$$
\begin{aligned}
(z ; q)_{\infty} & =\exp \left(\frac{-1}{q^{\ell^{2}}-1} \cdot \int_{0}^{z^{\ell}} \frac{\log (1-t)}{t} d t\right) \cdot \prod_{k=0}^{\ell-1}\left(1-\varepsilon^{k} z\right)^{k / \ell} \cdot(1+\mathcal{O}(q-\varepsilon))= \\
& =\exp \left(\frac{1}{q^{\ell^{2}}-1} \sum_{n=1}^{\infty} \frac{1}{n^{2}} \cdot z^{\ell n}\right) \cdot \prod_{k=0}^{\ell-1}\left(1-\varepsilon^{k} z\right)^{k / \ell} \cdot(1+\mathcal{O}(q-\varepsilon)) .
\end{aligned}
$$

Proof. Taylor expansion of $\log (1-t)$ shows that the two expressions in right-hand-side of (2.1) are equivalent. Now, the function $(z ; q)_{\infty}$ satisfies the difference equation

$$
\left(z q^{\ell} ; q\right)_{\infty}=\frac{1}{(z ; q)_{\ell}}(z ; q)_{\infty}
$$

and it is uniquely determined by this property along with the condition $(0 ; q)_{\infty}=1$. But

$$
\psi_{z}(q):=\exp \left(\frac{1}{q^{\ell^{2}}-1} \sum_{n=1}^{\infty} \frac{1}{n^{2}} z^{\ell n}\right) \cdot \prod_{k=0}^{\ell-1}\left(1-\varepsilon^{k} z\right)^{k / \ell}
$$

has the asymptotic behavior, for $q \rightarrow \varepsilon$, of the solution of (2.2); in fact we have

$$
\begin{gathered}
\frac{\psi_{z}(q)}{(z ; q)_{\ell} \cdot \psi_{z q^{\ell}}(q)}= \\
=\frac{\exp \left(\left(q^{\ell^{2}}-1\right)^{-1} \cdot \sum_{n=1}^{\infty} \frac{1}{n^{2}} z^{\ell n}\right) \cdot \prod_{k=0}^{\ell-1}\left(1-\varepsilon^{k} z\right)^{k / \ell}}{\prod_{k=0}^{\ell-1}\left(1-z q^{k}\right) \cdot \exp \left(\left(q^{\ell^{2}}-1\right)^{-1} \sum_{n=1}^{\infty} \frac{1}{n^{2}} q^{\ell^{2} n} z^{\ell n}\right) \cdot \prod_{k=0}^{\ell-1}\left(1-\varepsilon^{k} z q^{\ell}\right)^{k / \ell}}= \\
=\frac{\prod_{k=0}^{\ell-1}\left(1-\varepsilon^{k} z\right)^{k / \ell}}{\prod_{k=0}^{\ell-1}\left(1-\varepsilon^{k} z q^{\ell}\right)^{k / \ell} \cdot \prod_{k=0}^{\ell-1}\left(1-z q^{k}\right)} \cdot \exp \left(\left(q^{\ell^{2}}-1\right)^{-1} \cdot \sum_{n=1}^{\infty} \frac{\left(1-q^{\ell^{2} n}\right)}{n^{2}} z^{\ell n}\right)= \\
=\prod_{k=0}^{\ell-1}\left(\frac{1-\varepsilon^{k} z}{1-\varepsilon^{k} z q^{\ell}}\right)^{k / \ell} \cdot \frac{1}{\prod_{k=0}^{\ell-1}\left(1-z q^{k}\right)} \cdot \exp \left(-\sum_{n=1}^{\infty} \frac{q^{\ell^{2} n}-1}{q^{\ell^{2}}-1} \cdot \frac{\left(z^{\ell}\right)^{n}}{n^{2}}\right)= \\
=\prod_{k=0}^{\ell-1}\left(\frac{1-\varepsilon^{k} z}{1-\varepsilon^{k} z q^{\ell}}\right)^{k / \ell} \cdot \frac{1}{\prod_{k=0}^{\ell-1}\left(1-z q^{k}\right)} \cdot \exp \left(-\sum_{n=1}^{\infty}(n)_{q^{\ell^{2}}}\left(z^{\ell}\right)^{n} / n^{2}\right)
\end{gathered}
$$

when $q \longrightarrow \varepsilon$ we have $\lim _{q \rightarrow \varepsilon}(n)_{q^{\ell^{2}}}=n, \lim _{q \rightarrow \varepsilon} \prod_{k=0}^{\ell-1}\left(\frac{1-\varepsilon^{k} z}{1-\varepsilon^{k} z q^{\ell}}\right)^{k / \ell}=1$, and $\lim _{q \rightarrow \varepsilon} \frac{1}{\prod_{k=0}^{\ell-1}\left(1-z q^{k}\right)}=\left(\prod_{k=0}^{\ell-1}\left(1-z \varepsilon^{k}\right)\right)^{-1}=\prod_{k=0}^{\ell-1} \varepsilon^{k} \cdot \prod_{k=0}^{\ell-1}\left(\varepsilon^{k}-z\right)=1-z^{\ell} ;$ thus $\lim _{q \rightarrow \varepsilon} \frac{\psi_{z}(q)}{(z ; q)_{\ell} \cdot \psi_{z q^{\ell}}(q)}=\frac{1}{1-z^{\ell}} \cdot \exp \left(-\sum_{n=1}^{\infty} \frac{1}{n}\left(z^{\ell}\right)^{n}\right)=\frac{\exp \left(\log \left(1-z^{\ell}\right)\right)}{1-z^{\ell}}=\frac{1-z^{\ell}}{1-z^{\ell}}=1$, i. e. $\lim _{q \rightarrow 1} \frac{\psi_{z}(q)}{(z ; q)_{\ell} \cdot \psi_{z q} \ell(q)}=1$. Moreover from definition $\psi_{0}(q)=1$. The claim follows. 


\section{$\S 3$ Braiding of quantum enveloping algebras}

3.1. As we said, it is well known that quantum algebras $U_{h}(\mathfrak{g})$ are quasitriangular; this is proved by means of Drinfeld's method of the "quantum double" (cf. [Dr] and others). On the other hand, for the $\mathbb{C}(q)$-algebras $U_{q}^{M}(\mathfrak{g})$ the correct statement is that they are braided; for $\mathfrak{g}=\mathfrak{s l}(2)$, this is proved in [Re]: here we quickly perform the (straightforward) generalization.

To begin with we define a suitable completion of $U_{q}^{M}(\mathfrak{g})^{\otimes 2}$, namely

$$
U_{q}^{M}(\mathfrak{g})^{\widehat{\otimes} 2}:=\left\{\sum_{n=0}^{+\infty} \mathcal{E}_{n} \cdot P_{n}^{-} \otimes P_{n}^{+} \cdot \mathcal{F}_{n}\right\}
$$

where $P_{n}^{-} \in U_{q}^{M}\left(\mathfrak{b}_{-}\right), P_{n}^{+} \in U_{q}^{M}\left(\mathfrak{b}_{+}\right)\left(U_{q}^{M}\left(\mathfrak{b}_{ \pm}\right)\right.$being opposite quantum Borel subalgebras), $\mathcal{E}_{n} \in \sum_{|\beta|=n}\left(U_{q}^{M}(\mathfrak{g})\right)_{\beta}, \mathcal{F}_{n} \in \sum_{|\beta|=-n}\left(U_{q}^{M}(\mathfrak{g})\right)_{\beta}$. It is clear that $U_{q}^{M}(\mathfrak{g})^{\widehat{\otimes} 2}$ is a completion of $U_{q}^{M}(\mathfrak{g})^{\otimes 2}$ as Hopf algebra. From now on, as in [DD], [DP], we set $E_{\alpha}:=X_{\alpha}, F_{\alpha}:=Y_{\alpha}$.

Theorem 3.2. Let $\mathcal{R}^{(0)}$ be the algebra automorphism of $U_{q}^{M}(\mathfrak{g})^{{ }^{\otimes} 2}$ defined by

$$
\begin{aligned}
\mathcal{R}^{(0)}\left(L_{\mu} \otimes 1\right):=L_{\mu} \otimes 1, & \mathcal{R}^{(0)}\left(1 \otimes L_{\mu}\right):=1 \otimes L_{\mu} \\
\mathcal{R}^{(0)}\left(E_{i} \otimes 1\right):=E_{i} \otimes L_{-\alpha_{i}}, & \mathcal{R}^{(0)}\left(1 \otimes E_{i}\right):=L_{-\alpha_{i}} \otimes E_{i} \\
\mathcal{R}^{(0)}\left(F_{i} \otimes 1\right):=F_{i} \otimes L_{\alpha_{i}}, & \mathcal{R}^{(0)}\left(1 \otimes F_{i}\right):=L_{\alpha_{i}} \otimes F_{i}
\end{aligned}
$$

$(i=1, \ldots, n ; \mu \in M)$ and let $R^{(1)} \in U_{q}^{M}(\mathfrak{g})^{\widehat{\otimes} 2}$ be defined by

$$
R^{(1)}:=\prod_{\alpha \in R^{+}} \exp _{q_{\alpha}}\left(\left(q_{\alpha}^{-1}-q_{\alpha}\right) E_{\alpha} \otimes F_{\alpha}\right)
$$

Then $\left(U_{q}^{M}(\mathfrak{g}), \operatorname{Ad}\left(R^{(1)}\right) \circ \mathcal{R}^{(0)}\right)$ is a braided Hopf algebra, with $R^{(1)}$ as $R$-matrix.

Proof. We just outline the main steps, details being trivial. First of all, direct computation on generators shows that (1.9) and (1.10) hold. Then define $C \in U_{q}^{Q}(\mathfrak{g})^{\widehat{\otimes} 2}\left(\subset U_{q}^{M}(\mathfrak{g})^{\widehat{\otimes} 2}\right)$ by

$$
C:=\sum_{\beta \in Q_{+}} q^{(\beta, \beta)} \cdot\left(K_{\beta}^{-1} \otimes K_{\beta}\right) \cdot C_{\beta}
$$

where $Q_{+}:=Q \cap P_{+}$is the positive root lattice and $C_{\beta}$ is the canonical element of the bilinear pairing $\left(U_{q}^{Q}\left(\mathfrak{b}_{+}\right)\right)_{\beta} \times\left(U_{q}^{Q}\left(\mathfrak{b}_{-}\right)\right)_{-\beta} \longrightarrow \mathbb{C}(q)$ among quantum Borel algebras; let also $\Phi:=\mathcal{R}^{(0)^{-1}}$; then it is proved in [Ta], Theorem 4.3.3 that $\left(U_{q}^{Q}(\mathfrak{g}), C, \Phi\right)$ is a pretriangular Hopf algebra; the same proof also works for $U_{q}^{M}(\mathfrak{g})$ instead of $U_{q}^{Q}(\mathfrak{g})$. Now trivial checking yields $R^{(1)}=\Phi^{-1}(C)$ (using Proposition 3.7 in [DD]); therefore $\left(U_{q}^{M}(\mathfrak{g}), C, \Phi\right)$ being pretriangular implies that $\left(U_{q}^{M}(\mathfrak{g}), \operatorname{Ad}\left(R^{(1)}\right) \circ \mathcal{R}^{(0)}\right)$ is braided. 
Remark 3.3. Applying the remarks in $\S 2$ we can provide a multiplicative formula for the universal $R$-matrix $R^{(1)}$ of $U_{q}^{M}(\mathfrak{g})$, namely

$$
\begin{gathered}
R^{(1)}=\prod_{\alpha \in R^{+}} \exp _{q_{\alpha}}\left(\left(q_{\alpha}{ }^{-1}-q_{\alpha}\right) \cdot E_{\alpha} \otimes F_{\alpha}\right)= \\
=\prod_{\alpha \in R^{+}} e_{q_{\alpha}^{2}}\left(\left(q_{\alpha}{ }^{-1}-q_{\alpha}\right) \cdot\left(1-q_{\alpha}^{2}\right) \cdot E_{\alpha} \otimes F_{\alpha}\right)= \\
=\prod_{\alpha \in R^{+}} e_{q_{\alpha}^{2}}\left(\left(q_{\alpha}{ }^{-1}-q_{\alpha}\right) \cdot q_{\alpha}\left(q_{\alpha}^{-1}-q_{\alpha}\right) \cdot E_{\alpha} \otimes F_{\alpha}\right)= \\
=\prod_{\alpha \in R^{+}} e_{q_{\alpha}^{2}}\left(q_{\alpha} \bar{E}_{\alpha} \otimes \bar{F}_{\alpha}\right)=\prod_{\alpha \in R^{+}}\left(q_{\alpha} \cdot \bar{E}_{\alpha} \otimes \bar{F}_{\alpha} ; q_{\alpha}^{2}\right)_{\infty}^{-1}
\end{gathered}
$$

where $\bar{E}_{\alpha}:=\left(q_{\alpha}-q_{\alpha}^{-1}\right) E_{\alpha}$ and $\bar{F}_{\alpha}:=\left(q_{\alpha}-q_{\alpha}^{-1}\right) F_{\alpha}$ denote modified root vectors; in other words

$$
R^{(1)}=\prod_{\alpha \in R^{+}}\left(q_{\alpha} \cdot \bar{E}_{\alpha} \otimes \bar{F}_{\alpha} ; q_{\alpha}^{2}\right)_{\infty}^{-1} .
$$

Definition 3.4. We let $\mathcal{U}_{q}^{M}(\mathfrak{g})$ be the $\mathbb{C}\left[q, q^{-1}\right]$-subalgebra of $U_{q}^{M}(\mathfrak{g})$ generated by

$$
\left\{\bar{F}_{\alpha}, L_{\mu}, \bar{E}_{\alpha} \mid \alpha \in R^{+}, \mu \in M\right\} .
$$

Furthermore, for any $c \in \mathbb{C}$ we let

$$
\mathcal{U}_{c}^{M}(\mathfrak{g}):=\mathcal{U}_{q}^{M}(\mathfrak{g}) /(q-c) \mathcal{U}_{q}^{M}(\mathfrak{g}) \cong \mathcal{U}_{q}^{M}(\mathfrak{g}) \otimes_{\mathbb{C}\left[q, q^{-1}\right]} \mathbb{C}
$$

(with $\mathbb{C} \cong \mathbb{C}\left[q, q^{-1}\right] /(q-c)$ ) be the corresponding specialized algebra.

Remark 3.5. The previous definition is different but equivalent to the original one in [DP], $\S 12$, equivalence arising from the very description of $\mathcal{U}_{q}^{M}(\mathfrak{g})$ made therein. It is also proved in $[\mathrm{DP}]$ that $\mathcal{U}_{q}^{M}(\mathfrak{g})$ is a $\mathbb{C}\left[q, q^{-1}\right]$-integer form of $U_{q}^{M}(\mathfrak{g})$.

3.6. Our goal now is to show that $\mathcal{U}_{q}^{M}(\mathfrak{g})$ is braided: to be precise, we could say that the braiding structure of $U_{q}^{M}(\mathfrak{g})$ gives by restriction a braiding structure for $\mathcal{U}_{q}^{M}(\mathfrak{g})$. To begin with, we define a suitable completion of $\mathcal{U}_{q}^{M}(\mathfrak{g})^{\otimes 2}$ (mimicking §3.1), namely

$$
\mathcal{U}_{q}^{M}(\mathfrak{g}){ }^{\widehat{\otimes} 2}:=\left\{\sum_{n=0}^{+\infty} \overline{\mathcal{E}}_{n} \cdot P_{n}^{-} \otimes P_{n}^{+} \cdot \overline{\mathcal{F}}_{n}\right\}
$$

where $P_{n}^{-} \in \mathcal{U}_{q}^{M}\left(\mathfrak{b}_{-}\right), P_{n}^{+} \in \mathcal{U}_{q}^{M}\left(\mathfrak{b}_{+}\right), \overline{\mathcal{E}}_{n} \in \sum_{|\beta|=n}\left(\mathcal{U}_{q}^{M}(\mathfrak{g})\right)_{\beta}, \overline{\mathcal{F}}_{n} \in \sum_{|\beta|=-n}\left(\mathcal{U}_{q}^{M}(\mathfrak{g})\right)_{\beta}$. It is clear that $\mathcal{U}_{q}^{M}(\mathfrak{g})^{\widehat{\otimes} 2}$ is a completion of $\mathcal{U}_{q}^{M}(\mathfrak{g})^{\otimes 2}$ as Hopf algebra; moreover we have $\mathcal{U}_{q}^{M}(\mathfrak{g})^{\widehat{\otimes} 2} \subseteq U_{q}^{M}(\mathfrak{g})^{\widehat{\otimes} 2}$ via the natural embedding $\mathcal{U}_{q}^{M}(\mathfrak{g}) \longleftrightarrow U_{q}^{M}(\mathfrak{g})$. 
Proposition 3.7. The restriction of $\mathcal{R}^{(0)}$ (cf. Corollary 3.3) to $\mathcal{U}_{q}^{M}(\mathfrak{g})^{\widehat{\otimes} 2}$ is defined by

$$
\begin{array}{cl}
\widetilde{\mathcal{R}^{(0)}}\left(L_{\mu} \otimes 1\right):=L_{\mu} \otimes 1, & \widetilde{\mathcal{R}^{(0)}}\left(1 \otimes L_{\mu}\right):=1 \otimes L_{\mu} \\
\widetilde{\mathcal{R}^{(0)}}\left(\bar{E}_{\alpha} \otimes 1\right):=\bar{E}_{\alpha} \otimes L_{-\alpha}, & \widetilde{\mathcal{R}^{(0)}}\left(1 \otimes \bar{E}_{\alpha}\right):=L_{-\alpha} \otimes \bar{E}_{i} \\
\widetilde{\mathcal{R}^{(0)}}\left(\bar{F}_{\alpha} \otimes 1\right):=\bar{F}_{\alpha} \otimes L_{\alpha}, & \widetilde{\mathcal{R}^{(0)}}\left(1 \otimes \bar{F}_{\alpha}\right):=L_{\alpha} \otimes \bar{F}_{\alpha}
\end{array}
$$

$\left(\mu \in M, \alpha \in R^{+}\right)$so that $\mathcal{R}^{(0)}$ restricts to an algebra automorphism $\widetilde{\mathcal{R}^{(0)}}$ of $\mathcal{U}_{q}^{M}(\mathfrak{g})^{\widehat{\otimes} 2}$. Moreover, let $R^{(1)} \in U_{q}^{M}(\mathfrak{g})^{\widehat{\otimes} 2}$ be defined (as in Corollary 3.3) by

$$
R^{(1)}:=\prod_{\alpha \in R^{+}} \exp _{q_{\alpha}}\left(\left(q_{\alpha}^{-1}-q_{\alpha}\right) E_{\alpha} \otimes F_{\alpha}\right)=\prod_{\alpha \in R^{+}}\left(q_{\alpha} \cdot \bar{E}_{\alpha} \otimes \bar{F}_{\alpha} ; q_{\alpha}^{2}\right)_{\infty}^{-1}
$$

Then $\operatorname{Ad}\left(R^{(1)}\right)$ restricts to an automorphism $\widetilde{\mathcal{R}^{(1)}}$ of $\mathcal{U}_{q}^{M}(\mathfrak{g})^{\widehat{\otimes} 2}$, and $\left(\mathcal{U}_{q}^{M}(\mathfrak{g}), \widetilde{\mathcal{R}}\right)$ with $\widetilde{\mathcal{R}}:=\widetilde{\mathcal{R}^{(1)}} \circ \widetilde{\mathcal{R}^{(0)}}$ - is a braided Hopf algebra.

Proof. The first part of the statement is trivial. As for the second, we must recall that the specialization $\mathcal{U}_{1}^{M}(\mathfrak{g}):=\mathcal{U}_{q}^{M}(\mathfrak{g}) /(q-1) \mathcal{U}_{q}^{M}(\mathfrak{g})$ is a commutative $\mathbb{C}$-algebra (cf. [DP], $\S 12)$. Now from (3.1) we have

$$
R^{(1)}=\prod_{\alpha \in R^{+}}\left(q_{\alpha} \cdot \bar{E}_{\alpha} \otimes \bar{F}_{\alpha} ; q_{\alpha}^{2}\right)_{\infty}^{-1}=\prod_{\alpha \in R^{+}} R_{\alpha}^{(1)}
$$

letting $R_{\alpha}^{(1)}:=\left(q_{\alpha} \cdot \bar{E}_{\alpha} \otimes \bar{F}_{\alpha} ; q_{\alpha}^{2}\right)_{\infty}^{-1}$ for all $\alpha \in R^{+}$, and Lemma 2.2 (for $\varepsilon=1$ ) gives

$$
R_{\alpha}^{(1)}=\exp \left(\frac{1}{q-1} \cdot \frac{1}{2 d_{\alpha}} \cdot \varphi\left(q_{\alpha} \cdot \bar{E}_{\alpha} \otimes \bar{F}_{\alpha}\right)\right) \cdot\left(1-q_{\alpha} \cdot \bar{E}_{\alpha} \otimes \bar{F}_{\alpha}\right)^{-1 / 2} \cdot(1+\mathcal{O}(q-1))
$$

where we set $\varphi(z):=\sum_{n=1}^{\infty} \frac{1}{n^{2}} z^{n}$, as usual. Therefore we fall within the framework of [Re], §3, hence we can apply Reshetikin's trick to conclude: namely, applying Lemma 3.2.2 of [Re] we get for all $\alpha \in R^{+}$

$$
\operatorname{Ad}\left(R_{\alpha}^{(1)}\right)(a)=R_{\alpha}^{(1)} \cdot a \cdot R_{\alpha}^{(1)}{ }^{-1} \in \mathcal{U}_{q}^{M}(\mathfrak{g})^{\widehat{\otimes} 2}
$$

for all $a \in \mathcal{U}_{q}^{M}(\mathfrak{g})^{\widehat{\otimes} 2}$, i. e. $\operatorname{Ad}\left(R_{\alpha}^{(1)}\right)$ restricts to an automorphism $\widetilde{\mathcal{R}_{\alpha}^{(1)}}$ of $\mathcal{U}_{q}^{M}(\mathfrak{g})^{\widehat{\otimes} 2}$; thus also $\operatorname{Ad}\left(R^{(1)}\right)=\operatorname{Ad}\left(\prod_{\alpha \in R^{+}} R_{\alpha}^{(1)}\right)=\prod_{\alpha \in R^{+}} \operatorname{Ad}\left(R_{\alpha}^{(1)}\right)=\prod_{\alpha \in R^{+}} \widetilde{\mathcal{R}_{\alpha}^{(1)}}$ does restrict to an automorphism $\widetilde{\mathcal{R}^{(1)}}$ of $\mathcal{U}_{q}^{M}(\mathfrak{g})^{\widehat{\otimes} 2}$ as claimed. Then Theorem 3.2 yields the claim.

Corollary 3.8. For any $c \in \mathbb{C}$, let $\mathcal{R}_{c}$ be the algebra automorphism of $\mathcal{U}_{c}^{M}(\mathfrak{g})^{\widehat{\otimes} 2}$ given by specialization of $\widetilde{\mathcal{R}}$ at $q=c$. Then $\left(\mathcal{U}_{c}^{M}(\mathfrak{g}), \mathcal{R}_{c}\right)$ is a braided Hopf algebra. 


\section{$\S 4$ The geometrical meaning of the braiding structure at roots of 1}

4.1 Geometric framework. In this section we turn to geometry: our aim is to show that the series describing the adjoint action of the $R$-matrix of a quantum group are more than formal objects, for they do converge, in a proper sense, so that such action does yield well-defined automorphisms of geometric objects.

Let $G$ be a connected simply connected semisimple Poisson algebraic group over $\mathbb{C}$ with $\mathfrak{g}$ as tangent Lie bialgebra; then there exists a uniquely defined connected simply connected semisimple affine algebraic Poisson group $H$ over $\mathbb{C}$ with tangent Lie bialgebra $\mathfrak{g}^{*}$ and algebra of polynomial functions $F[H]$, which is called the Poisson group dual of $G$ (cf. e. g. $[\mathrm{DP}], \S 11)$.

Let $\ell \in \mathbb{N}$ be odd, $\ell>d:=\max _{i}\left\{d_{i}\right\}$, or $\ell=1$; then let $\varepsilon \in \mathbb{C}$ be a primitive $\ell$-th root of 1 . As a matter of notation, let $U_{\varepsilon}:=\mathcal{U}_{\varepsilon}^{P}(\mathfrak{g}), Z_{\varepsilon}:=Z\left(U_{\varepsilon}\right)$ (the centre of $\left.U_{\varepsilon}\right)$. Everything in the sequel can then be suitably extended to the case of quantum group $U_{q}^{M}(\mathfrak{g})$ with general lattice $M$. From the analysis in $[\mathrm{DP}]$ (cf. also [DK], [DKP]) we recall the following results:

(a) The subalgebra $Z_{0}$ of $U_{\varepsilon}$ generated by $\bar{E}_{\alpha}^{\ell}, \bar{F}_{\alpha}^{\ell}, L_{i}^{\ell}\left(\alpha \in R^{+}, i=1, \ldots, n\right)$ is central, i. e. $Z_{0} \subseteq Z_{\varepsilon}$.

(b) $Z_{\varepsilon}$ and $Z_{0}$ inherit (from $\mathcal{U}_{q}^{P}(\mathfrak{g})$ ) canonical structures of Poisson algebras; in particular, $Z_{0}$ is a Poisson Hopf algebra.

(c) There exists an isomorphism $Z_{0} \cong F[H]$ as Poisson Hopf algebras (with respect to a suitable normalization of the Poisson bracket on $\left.Z_{0}\right)$, hence $\operatorname{Spec}\left(Z_{0}\right) \cong H$ as Poisson (complex affine algebraic) groups. In particular (for $\ell=1) \operatorname{Spec}\left(\mathcal{U}_{1}^{P}(\mathfrak{g})\right) \cong H$.

Recall that in $[\mathrm{DK}],[\mathrm{DKP}],[\mathrm{DP}]$ the spectra $\operatorname{Spec}\left(U_{\varepsilon}\right), \operatorname{Spec}\left(Z_{\varepsilon}\right)$, and $\operatorname{Spec}\left(Z_{0}\right)$ are introduced as the set of isomorphism classes of finite dimensional representations of the corresponding algebras $U_{\varepsilon}, Z_{\varepsilon}$, and $Z_{0}$; in particular $\operatorname{Spec}\left(Z_{\varepsilon}\right)$ and $\operatorname{Spec}\left(Z_{0}\right)$ can be identified with usual geometric objects, namely complex affine algebraic varieties describing the maximal spectrum of $Z_{\varepsilon}$ and $Z_{0}$; since $Z_{0} \cong F[H]$ as Poisson Hopf algebras, we also have $\operatorname{Spec}\left(Z_{0}\right) \cong H$ as Poisson affine algebraic groups (over $\mathbb{C}$ ); thus in the sequel we will also set $H_{\varepsilon}:=\operatorname{Spec}\left(Z_{\varepsilon}\right)$ and $S_{\varepsilon}:=\operatorname{Spec}\left(U_{\varepsilon}\right)$. The analysis in $[\mathrm{DP}] \operatorname{describes} \operatorname{Spec}\left(U_{\varepsilon}\right)$ as (espace étalé of) a sheaf — or a fibre bundle — of algebras over $\operatorname{Spec}\left(Z_{0}\right)$ or $\operatorname{Spec}\left(Z_{\varepsilon}\right)$; in particular we can think at $U_{\varepsilon}$ as the algebra of global sections of this sheaf.

Now set

$$
y_{\alpha}:=\left.\bar{F}_{\alpha}^{\ell}\right|_{q=\varepsilon}, \quad z_{\lambda}:=\left.L_{\lambda}^{\ell}\right|_{q=\varepsilon}, \quad x_{\alpha}:=\left.\bar{E}_{\alpha}^{\ell}\right|_{q=\varepsilon} \quad \forall \alpha \in R^{+}, \lambda \in P
$$

and in particular $y_{i}:=y_{\alpha_{i}}, z_{i}:=z_{\omega_{i}}, x_{i}:=x_{\alpha_{i}}(i=1, \ldots, n)$. Following [DK], §3.5, we denote by $\widehat{Z}_{0}$ the algebra of all formal power series in the $y_{\alpha}$ 's, $z_{i}^{ \pm 1}$ 's, $x_{\alpha}$ 's which converge to meromorphic functions for all complex values of the $y_{\alpha}$ 's, $x_{\alpha}$ 's, and all non-zero complex values of the $z_{i}$ 's; then let $\widehat{U}_{\varepsilon}:=\widehat{Z}_{0} \otimes_{Z_{0}} U_{\varepsilon}, \widehat{Z}_{\varepsilon}:=\widehat{Z}_{0} \otimes_{Z_{0}} Z_{\varepsilon}$. In other words we can think at $\widehat{U}_{\varepsilon}$ as the algebra of global meromorphic sections of the corresponding bundle of algebras over $\operatorname{Spec}\left(Z_{0}\right) \cong H$. Similar notations and definitions will be used when dealing with square tensor powers, like $Z_{0}{ }^{\otimes 2}, Z_{\varepsilon}{ }^{\otimes 2}$, and so on. Notice also that $\operatorname{Spec}\left(Z_{0} \otimes Z_{0}\right)=$ $\operatorname{Spec}\left(Z_{0}\right) \times \operatorname{Spec}\left(Z_{0}\right)=H \times H, \operatorname{Spec}\left(Z_{\varepsilon} \otimes Z_{\varepsilon}\right)=\operatorname{Spec}\left(Z_{\varepsilon}\right) \times \operatorname{Spec}\left(Z_{\varepsilon}\right)=H_{\varepsilon} \times H_{\varepsilon}$, $\operatorname{Spec}\left(U_{\varepsilon} \otimes U_{\varepsilon}\right)=\operatorname{Spec}\left(U_{\varepsilon}\right) \times \operatorname{Spec}\left(U_{\varepsilon}\right)=S_{\varepsilon} \times S_{\varepsilon}$, 
Warning: when dealing with cross-product spaces like $X \times Y$, we shall use left subscripts to denote functions of either of the two spaces, viz. ${ }_{2} x:=1 \otimes x,{ }_{1} \bar{E}_{\alpha}:=\bar{E}_{\alpha} \otimes 1$, etc.

Let $\mathcal{H}^{(N)}$ be any fixed ramified $N$-fold covering (for $N \in \mathbb{N} \cup\{\infty\}$ ) of $H$ (so that $\mathcal{H}^{(N)} \times$ $\mathcal{H}^{(N)}$ is an $N$-fold covering of $\left.H \times H\right)$; then we denote by $\mathcal{H}_{\varepsilon}^{(N)}$ and $\mathcal{S}_{\varepsilon}^{(N)}$ the fiber products $\mathcal{H}_{\varepsilon}^{(N)}:=\mathcal{H}^{(N)} \times_{H} H_{\varepsilon}$ and $\mathcal{S}_{\varepsilon}^{(N)}:=\mathcal{H}^{(N)} \times_{H} S_{\varepsilon}$. Notice that $\mathcal{H}_{\varepsilon}^{(N)}=\operatorname{Spec}\left(\widehat{Z}_{\varepsilon}\right)$ and $\mathcal{S}_{\varepsilon}^{(N)}=\operatorname{Spec}\left(\widehat{U}_{\varepsilon}\right)$; furthermore, $\mathcal{H}^{(N)}$ and $\mathcal{H}_{\varepsilon}^{(N)}$ clearly have a unique Poisson structure compatible with the covering map, so that $\mathcal{H}_{\varepsilon}^{(N)}$ is a (complex analytic) Poisson variety and $\mathcal{H}^{(N)}$ is a (complex analytic) Poisson group. Finally, $\tau:=\sigma^{*}: H \times H \longrightarrow H \times H$ ( $\sigma$ being defined in $\S 1.3)$ is given by $(x, y) \mapsto(y, x)$; then $\tau^{(N)}: \mathcal{H}^{(N)} \times \mathcal{H}^{(N)} \longrightarrow \mathcal{H}^{(N)} \times \mathcal{H}^{(N)}$, also given by $(x, y) \mapsto(y, x)$, is a lifting of $\tau$ to $\mathcal{H}^{(N)} \times \mathcal{H}^{(N)}$.

Fix now $\ell>1$ : we are ready for the next result, which claims that the "formal automorphism" $\mathcal{R}_{\varepsilon}$ giving the braiding structure of $U_{\varepsilon}$ actually does converge in a proper sense.

Proposition 4.2. The algebra automorphism $\mathcal{R}_{\varepsilon}: U_{\varepsilon}^{\widehat{\otimes} 2} \rightarrow U_{\varepsilon}^{\widehat{\otimes} 2}$ defines a meromorphic automorphism $\mathcal{R}_{\varepsilon, \infty}^{*}$ of $\mathcal{S}_{\varepsilon}^{(\infty)} \times \mathcal{S}_{\varepsilon}^{(\infty)}$, which restricts to meromorphic Poisson automorphisms $\mathcal{R}_{\varepsilon, \infty}^{*}: \mathcal{H}_{\varepsilon}^{(\infty)} \times \mathcal{H}_{\varepsilon}^{(\infty)} \longrightarrow \mathcal{H}_{\varepsilon}^{(\infty)} \times \mathcal{H}_{\varepsilon}^{(\infty)}$ and $\mathcal{R}_{\varepsilon, \infty}^{*}: \mathcal{H}^{(\infty)} \times \mathcal{H}^{(\infty)} \longrightarrow \mathcal{H}^{(\infty)} \times \mathcal{H}^{(\infty)}$.

Moreover, $\mathcal{R}_{\varepsilon, \infty}^{*}$ and its restrictions enjoy the dual properties of (1.4-6); in particular, $\mathcal{R}_{\varepsilon, \infty}^{*} \neq \tau$, and $m\left(\mathcal{R}_{\mathcal{H}}^{*}(x, y)\right)=m(y, x)=y \cdot x$ for all $x, y \in \mathcal{H}^{(\infty)}$ ( $m$ and ". " denoting the product of $\left.\mathcal{H}^{(\infty)}\right)$, and a braid group action exists on $\times$-powers of $\mathcal{H}^{(\infty)}$.

Proof. The first step in the proof amounts to show that series $\mathcal{R}_{\varepsilon}(x \otimes y)$ do converge almost everywhere on a suitable covering $\mathcal{S}_{\varepsilon}^{(\infty)} \times \mathcal{S}_{\varepsilon}^{(\infty)}$. Recall (cf. Proposition 3.2 and its proof) that $\widetilde{\mathcal{R}}:=\widetilde{\mathcal{R}^{(1)}} \circ \widetilde{\mathcal{R}^{(0)}}$, thus $\mathcal{R}_{\varepsilon}:=\mathcal{R}^{(1)}{ }_{\varepsilon} \circ \mathcal{R}^{(0)}{ }_{\varepsilon}$ with $\mathcal{R}^{(0)}{ }_{\varepsilon}:=\widetilde{\mathcal{R}^{(0)}} \bmod (q-\varepsilon)$ and $\mathcal{R}^{(1)}{ }_{\varepsilon}:=\widetilde{\mathcal{R}^{(1)}} \bmod (q-\varepsilon)$. For $\mathcal{R}^{(0)}{ }_{\varepsilon}$ the very definition implies that no problem of convergence (nor of domain of definition) occurs. For $\mathcal{R}_{\varepsilon}^{(1)}$, recall that $\widetilde{\mathcal{R}^{(1)}}:=\left.\operatorname{Ad}\left(R^{(1)}\right)\right|_{\mathcal{U}_{q}^{P(\mathfrak{g})} \widehat{\otimes}^{2}}$ and

$$
R^{(1)}:=\prod_{\alpha \in R^{+}}\left(q_{\alpha} \cdot \bar{E}_{\alpha} \otimes \bar{F}_{\alpha} ; q_{\alpha}^{2}\right)_{\infty}^{-1}=\prod_{\alpha \in R^{+}} R_{\alpha}^{(1)}
$$

where $R_{\alpha}^{(1)}:=\left(q_{\alpha} \cdot \bar{E}_{\alpha} \otimes \bar{F}_{\alpha} ; q_{\alpha}^{2}\right)_{\infty}^{-1}$, like in the proof of Proposition 3.2 ; therefore

$$
\operatorname{Ad}\left(R^{(1)}\right)=\prod_{\alpha \in R^{+}} \operatorname{Ad}\left(R_{\alpha}^{(1)}\right)=\prod_{\alpha \in R^{+}} \operatorname{Ad}\left(\left(q_{\alpha} \cdot \bar{E}_{\alpha} \otimes \bar{F}_{\alpha} ; q_{\alpha}^{2}\right)_{\infty}^{-1}\right) .
$$

Now again we apply Reshetikin's trick: from [Re], Lemma 3.2.2 and formulas (3.2.1011 ), and from our Lemma 2.2 we get

$$
\begin{aligned}
& \operatorname{Ad}\left(R_{\alpha}^{(1)}\right) \bmod (q-\varepsilon)=\operatorname{Ad}\left(\left(q_{\alpha} \cdot \bar{E}_{\alpha} \otimes \bar{F}_{\alpha} ; q_{\alpha}^{2}\right)_{\infty}^{-1}\right) \bmod (q-\varepsilon)= \\
& \quad=\operatorname{Ad}\left(\exp \left(\frac{\Phi_{\alpha}}{q-\varepsilon}\right)\right) \bmod (q-\varepsilon)=\exp \left(\operatorname{ad}_{\{,\}}\left(\Phi_{\alpha}\right)\right) \bmod (q-\varepsilon)= \\
& =\exp \left(\operatorname{ad}_{\{,\}}\left(\frac{\varepsilon}{2 d_{\alpha} \ell^{2}} \cdot \varphi\left(q_{\alpha} \bar{E}_{\alpha} \otimes \bar{F}_{\alpha}\right)\right)\right) \bmod (q-\varepsilon)
\end{aligned}
$$


whith

$$
\begin{gathered}
\Phi_{\alpha}:=\left(\frac{q-\varepsilon}{q_{\alpha}^{2 \ell^{2}}-1} \cdot \varphi\left(q_{\alpha} \bar{E}_{\alpha} \otimes \bar{F}_{\alpha}\right)-(q-\varepsilon) \cdot \log \left(\prod_{k=0}^{\ell-1} \frac{k}{\ell}\left(1-\varepsilon^{k} q_{\alpha} \bar{E}_{\alpha} \otimes \bar{F}_{\alpha}\right)\right)\right) . \\
\cdot(1+\mathcal{O}(q-\varepsilon))=\exp \left(\frac{q-\varepsilon}{q_{\alpha}^{2 \ell^{2}}-1} \cdot \varphi\left(q_{\alpha} \bar{E}_{\alpha} \otimes \bar{F}_{\alpha}\right)\right) \quad \bmod (q-\varepsilon)
\end{gathered}
$$

and

$$
\varphi(t):=\int_{0}^{t^{\ell}} \frac{\log (1-\tau)}{\tau} d \tau=\sum_{n=1}^{\infty} \frac{t^{\ell n}}{n^{2}} \quad \text { (by Taylor expansion) } .
$$

Notice that

$$
\begin{aligned}
\operatorname{ad}_{\{,\}}(t \cdot \varphi(x))(y)=\{t \cdot \varphi(x), y\} & =t \cdot\left\{\sum_{n=1}^{\infty} \frac{x^{\ell n}}{n^{2}}, y\right\}=t \cdot \sum_{n=1}^{\infty} \frac{1}{n^{2}} \cdot\left\{\left(x^{\ell}\right)^{n}, y\right\}= \\
& =t \cdot \sum_{n=1}^{\infty} \frac{1}{n^{2}} n\left(x^{\ell}\right)^{n-1} \cdot\left\{x^{\ell}, y\right\}=\sum_{n=1}^{\infty} \frac{\left(x^{\ell}\right)^{n-1}}{n} \cdot\left\{t \cdot x^{\ell}, y\right\}
\end{aligned}
$$

(because of Leibnitz' rule: $\{\cdot, y\}=-\operatorname{ad}_{\{,\}}(y)$ is a derivation!), hence

$$
\operatorname{ad}_{\{,\}}(t \cdot \varphi(x))=\psi\left(x^{\ell}\right) \cdot \operatorname{ad}_{\{,\}}\left(t \cdot x^{\ell}\right)
$$

with $\psi(t):=\frac{\log (1-y)}{y}=\sum_{n=0}^{\infty} \frac{y^{n}}{n+1}$ (by Taylor expansion again), and then

$$
\exp \left(\operatorname{ad}_{\{,\}}(t \cdot \varphi(x))\right)=\exp \left(\psi\left(x^{\ell}\right) \cdot \operatorname{ad}_{\{,\}}\left(t \cdot x^{\ell}\right)\right)
$$

together with (4.3) this gives

$$
\begin{aligned}
& \operatorname{Ad}\left(R_{\alpha}^{(1)}\right) \bmod (q-\varepsilon)=\exp \left(\operatorname{ad}_{\{,\}}\left(\frac{\varepsilon}{2 d_{\alpha} \ell^{2}} \cdot \varphi\left(q_{\alpha} \bar{E}_{\alpha} \otimes \bar{F}_{\alpha}\right)\right)\right)= \\
& =\exp \left(\psi\left(q_{\alpha}^{\ell} \bar{E}_{\alpha}^{\ell} \otimes \bar{F}_{\alpha}^{\ell}\right) \cdot \operatorname{ad}_{\{,\}}\left(\frac{\varepsilon}{2 d_{\alpha} \ell^{2}} q_{\alpha}^{\ell} \cdot \bar{E}_{\alpha}^{\ell} \otimes \bar{F}_{\alpha}^{\ell}\right)\right) \bmod (q-\varepsilon)= \\
& \quad=\exp \left(\frac{\varepsilon}{2 d_{\alpha} \ell^{2}} \psi\left(x_{\alpha} \otimes y_{\alpha}\right) \cdot \operatorname{ad}_{\{,\}}\left(x_{\alpha} \otimes y_{\alpha}\right)\right) \bmod (q-\varepsilon) .
\end{aligned}
$$

Therefore we have to show that the formal series

$$
\exp \left(\frac{\varepsilon}{2 d_{\alpha} \ell^{2}} \cdot \psi\left(x_{\alpha} \otimes y_{\alpha}\right) \cdot \operatorname{ad}_{\{,\}}\left(x_{\alpha} \otimes y_{\alpha}\right)\right)(x \otimes y)
$$

for $x, y$ generators of $U_{\varepsilon}$ (that is $x, y \in\left\{1, \bar{F}_{\alpha}, L_{i}, \bar{E}_{\alpha} \bmod (q-\varepsilon) \mid i=1, \ldots, n, \alpha \in\right.$ $\left.R^{+}\right\}$) does converge (to a meromorphic function on $\mathcal{S}_{\varepsilon}^{(\infty)} \times \mathcal{S}_{\varepsilon}^{(\infty)}$ ). But notice that the following obvious identity holds (for all $n \in \mathbb{N}$ )

$$
\left(\frac{\varepsilon}{2 d_{\alpha} \ell^{2}} \cdot \psi\left(x_{\alpha} \otimes y_{\alpha}\right) \cdot \operatorname{ad}_{\{,\}}\left(x_{\alpha} \otimes y_{\alpha}\right)\right)^{n}=\psi\left(x_{\alpha} \otimes y_{\alpha}\right)^{n} \cdot\left(\frac{\varepsilon}{2 d_{\alpha} \ell^{2}} \cdot \operatorname{ad}_{\{,\}}\left(x_{\alpha} \otimes y_{\alpha}\right)\right)^{n}
$$


because of Leibnitz' rule and $\operatorname{ad}_{\{,\}}\left(x_{\alpha} \otimes y_{\alpha}\right)\left(\frac{\varepsilon}{2 d_{\alpha} \ell^{2}} \psi\left(x_{\alpha} \otimes y_{\alpha}\right)\right)=0$; moreover, $\psi\left(x_{\alpha} \otimes y_{\alpha}\right)$ is a meromorphic function on the $\infty$-fold ramified covering $\mathcal{H}^{(\infty)} \times \mathcal{H}^{(\infty)}$ of $H \times H$. Now recall that

$$
[x \otimes y, z \otimes w]=[x, z] \otimes y w+x z \otimes[y, w] ;
$$

then set ${ }_{1} \mathrm{e}_{\alpha}:=\left.\operatorname{ad}\left[,{ }_{1}\right]\left({ }_{1} E_{\alpha}^{(\ell)}\right)\right|_{q=\varepsilon},{ }_{2} \mathrm{f}_{\alpha}:=\left.\operatorname{ad}_{[,,]}\left({ }_{2} F_{\alpha}^{(\ell)}\right)\right|_{q=\varepsilon}\left(X_{\alpha}^{(n)}:=\frac{X_{\alpha}^{n}}{[n]{ }_{q \alpha} !}\right)$, observe that

$$
\begin{aligned}
& { }_{1} \mathrm{e}_{\alpha}:=\left.\operatorname{ad}_{[,,]}\left({ }_{1} E_{\alpha}^{(\ell)}\right)\right|_{q=\varepsilon}=\left.\left(\frac{1}{q_{\alpha}^{\ell^{2}}-1} \cdot \operatorname{ad}_{[,,]}\left({ }_{1} \bar{E}_{\alpha}^{\ell}\right)\right)\right|_{q=\varepsilon}=\frac{\varepsilon}{2 d_{\alpha} \ell^{2}} \cdot \operatorname{ad}_{\{,\}}\left({ }_{1} x_{\alpha}\right) \\
& { }_{2} \mathrm{f}_{\alpha}:=\left.\operatorname{ad}_{[,,]}\left({ }_{2} F_{\alpha}^{(\ell)}\right)\right|_{q=\varepsilon}=\left.\left(\frac{1}{q_{\alpha}^{2 \ell^{2}}-1} \cdot \operatorname{ad}_{[,]}\left({ }_{2} \bar{F}_{\alpha}^{\ell}\right)\right)\right|_{q=\varepsilon}=\frac{\varepsilon}{2 d_{\alpha} \ell^{2}} \cdot \operatorname{ad}_{\{,\}}\left({ }_{2} y_{\alpha}\right)
\end{aligned}
$$

and let $m(x): y \mapsto x y$ (left multiplication by $x$ ); then formula (4.4) gives

$$
\frac{\varepsilon}{2 d_{\alpha} \ell^{2}} \cdot \operatorname{ad}_{\{,\}}\left(x_{\alpha} \otimes y_{\alpha}\right)={ }_{1} \mathrm{e}_{\alpha} \otimes m\left({ }_{2} y_{\alpha}\right)+m\left({ }_{1} x_{\alpha}\right) \otimes{ }_{2} \mathrm{f}_{\alpha} ;
$$

one trivially checks that ${ }_{1} \mathrm{e}_{\alpha} \otimes m\left({ }_{2} y_{\alpha}\right)$ and $m\left({ }_{1} x_{\alpha}\right) \otimes{ }_{2} \mathrm{f}_{\alpha}$ are operators which commute with each other, thus (4.5) gives

$$
\exp \left(\operatorname{ad}{ }_{\{,\}}\left(x_{\alpha} \otimes y_{\alpha}\right)\right)=\exp \left({ }_{1} \mathrm{e}_{\alpha} \otimes m\left({ }_{2} y_{\alpha}\right)\right) \circ \exp \left(m\left({ }_{1} x_{\alpha}\right) \otimes{ }_{2} \mathrm{f}_{\alpha}\right) ;
$$

for $x, y$ generators of $U_{\varepsilon}$ we have

$$
\begin{aligned}
& \exp \left(\psi\left(x_{\alpha} \otimes y_{\alpha}\right) \cdot m\left(x_{\alpha}\right) \otimes \mathrm{f}_{\alpha}\right)(x \otimes y)=\exp \left(\frac{\log \left(1-{ }_{1} x_{\alpha} \cdot{ }_{2} y_{\alpha}\right)}{{ }_{1} x_{\alpha} \cdot{ }_{2} y_{\alpha}} \cdot{ }_{1} x_{\alpha} \cdot \mathrm{f}_{\alpha}\right)(x \otimes y)= \\
& =\exp \left(\frac{\log \left(1-{ }_{1} x_{\alpha} \cdot{ }_{2} y_{\alpha}\right)}{{ }_{2} y_{\alpha}} \cdot \mathrm{f}_{\alpha}\right)(x \otimes y)=\exp \left(\frac{\log \left(1-{ }_{1} x_{\alpha} \cdot{ }_{2} y_{\alpha}\right)}{{ }_{2} y_{\alpha}} \cdot \mathrm{f}_{\alpha}\right)(y) \cdot x \otimes 1 .
\end{aligned}
$$

It is proved in $[\mathrm{DK}], \S 3$, that $\exp \left(t \cdot \mathrm{f}_{\alpha}\right)$ converges to a holomorphic automorphism of the algebra of global holomorphic sections of $S_{\varepsilon}$ (as a bundle over $H$ ), for all $t \in \mathbb{C}$; when $t$ is replaced with any meromorphic function on $H$, the series we get does converge to an automorphism of the algebra of meromorphic sections (cf. formulas in the proof of Proposition 3.5 of [DK]); since $\frac{\log \left(1-x_{1} x_{\alpha} \cdot 2 y_{\alpha}\right)}{2 y_{\alpha}}$ is meromorphic on the $\infty$-fold covering $\mathcal{H}^{(\infty)} \times \mathcal{H}^{(\infty)}$, we conclude that $\exp \left(\psi\left(x_{\alpha} \otimes y_{\alpha}\right) \cdot m\left({ }_{1} x_{\alpha}\right) \otimes{ }_{2} \mathrm{f}_{\alpha}\right)(x \otimes y)$ is a meromorphic section of $\mathcal{S}_{\varepsilon}^{(\infty)} \times \mathcal{S}_{\varepsilon}^{(\infty)}$; the same holds for $\exp \left(\psi\left(x_{\alpha} \otimes y_{\alpha}\right) \cdot{ }_{1} \mathrm{e}_{\alpha} \otimes m\left({ }_{2} y_{\alpha}\right)\right)$, and finally for $\left.\operatorname{Ad}\left(R_{\alpha}^{(1)}\right)\right|_{q=\varepsilon}=\left.\exp \left(\frac{\varepsilon}{2 d_{\alpha} \ell^{2}} \cdot \psi\left(x_{\alpha} \otimes y_{\alpha}\right) \cdot \operatorname{ad}_{\{,\}}\left(x_{\alpha} \otimes y_{\alpha}\right)\right)\right|_{q=\varepsilon}$, q. e. d.

For the second part, notice that $\mathcal{R}^{(0)}$ clearly leaves invariant both $\widehat{Z_{\varepsilon}{ }^{\otimes 2}}$ and $\widehat{Z_{0}{ }^{\otimes 2}}$, hence its dual leaves invariant $\mathcal{H}_{\varepsilon}^{(\infty)} \times \mathcal{H}_{\varepsilon}^{(\infty)}$ and $\mathcal{H}^{(\infty)} \times \mathcal{H}^{(\infty)} ;$ moreover, since $\mathcal{R}^{(1)} \varepsilon$ is a product of terms

$$
\left.\operatorname{Ad}\left(R_{\alpha}^{(1)}\right)\right|_{q=\varepsilon}=\left.\exp \left(\frac{-\varepsilon}{2 d_{\alpha} \ell^{2}} \cdot \psi\left(x_{\alpha} \otimes y_{\alpha}\right) \cdot \operatorname{ad}_{\{,\}}\left(x_{\alpha} \otimes y_{\alpha}\right)\right)\right|_{q=\varepsilon}
$$


since $\widehat{Z_{\varepsilon}^{\otimes 2}}$ and $\widehat{Z_{0}{ }^{\otimes 2}}$ are closed for the Poisson bracket, and since $x_{\alpha} \otimes y_{\alpha} \in \widehat{Z_{0}{ }^{\otimes 2}} \subseteq$ $\widehat{Z_{\varepsilon}{ }^{\otimes 2}}$, we have that the dual of $\mathcal{R}_{\varepsilon}^{(1)}$ leaves $\mathcal{H}_{\varepsilon}^{(\infty)} \times \mathcal{H}_{\varepsilon}^{(\infty)}$ and $\mathcal{H}^{(\infty)} \times \mathcal{H}^{(\infty)}$ invariant; thus we conclude that $\mathcal{R}_{\varepsilon}^{*}$ leaves $\mathcal{H}_{\varepsilon}^{(\infty)} \times \mathcal{H}_{\varepsilon}^{(\infty)}$ and $\mathcal{H}^{(\infty)} \times \mathcal{H}^{(\infty)}$ invariant. Finally, it clearly preserves the Poisson structure because $\mathcal{R}_{\varepsilon}$ is defined by specializing an algebra automorphism of $\widehat{\mathcal{U}_{q}^{P(\mathfrak{g})^{\otimes 2}}}$, whence

$$
\mathcal{R}_{\varepsilon}\left(\left\{x_{0}, y_{0}\right\}\right)=\left.\widetilde{\mathcal{R}}\left(\frac{[x, y]}{q-\varepsilon}\right)\right|_{q=\varepsilon}=\left.\frac{[\widetilde{\mathcal{R}}(x), \widetilde{\mathcal{R}}(y)]}{q-\varepsilon}\right|_{q=\varepsilon}=\left\{\mathcal{R}_{\varepsilon}\left(x_{0}\right), \mathcal{R}_{\varepsilon}\left(y_{0}\right)\right\} .
$$

The proof of the last part of the statement is completely trivial, by functoriality.

A deeper analysis yelds to improve the previous result, proving that the convergence already holds on finite ramified coverings, as the following shows.

Theorem 4.3. The meromorphic automorphism $\mathcal{R}_{\varepsilon}^{*}: \mathcal{S}_{\varepsilon}^{(\infty)} \times \mathcal{S}_{\varepsilon}^{(\infty)} \longrightarrow \mathcal{S}_{\varepsilon}^{(\infty)} \times \mathcal{S}_{\varepsilon}^{(\infty)}$ pushes down to a birational automorphism $\mathcal{R}_{\varepsilon, \ell}^{*}: \mathcal{S}_{\varepsilon}^{(2 \ell)} \times \mathcal{S}_{\varepsilon}^{(2 \ell)} \longrightarrow \mathcal{S}_{\varepsilon}^{(2 \ell)} \times \mathcal{S}_{\varepsilon}^{(2 \ell)} ;$ moreover, $\mathcal{R}_{\varepsilon, \ell}^{*} \neq$ $\tau^{(2 \ell)}$, and $\mathcal{R}_{\varepsilon, \ell}^{*}$ enjoys the dual properties of $(1.4-6)$.

The same holds with $H_{\varepsilon}$, resp. $H$ instead of $S_{\varepsilon}$, with a birational Poisson automorphism $\mathcal{R}_{\varepsilon, \ell}^{*}: \mathcal{H}_{\varepsilon}^{(2 \ell)} \times \mathcal{H}_{\varepsilon}^{(2 \ell)} \rightarrow \mathcal{H}_{\varepsilon}^{(2 \ell)} \times \mathcal{H}_{\varepsilon}^{(2 \ell)}$, resp. $\mathcal{R}_{\varepsilon, \ell}^{*}: \mathcal{H}^{(2 \ell)} \times \mathcal{H}^{(2 \ell)} \rightarrow \mathcal{H}^{(2 \ell)} \times \mathcal{H}^{(2 \ell)}:$ in particular, $m\left(\mathcal{R}_{\varepsilon, \ell}^{*}(x, y)\right)=m(y, x)=y \cdot x$ for all $x, y \in \mathcal{H}^{(2 \ell)}$ (where $m$ and ". "denote the product of $\left.\mathcal{H}^{(2 \ell)}\right)$, and a braid group action exists on $\times$-powers of $\mathcal{H}^{(2 \ell)}$.

Proof. It is clear that for $\mathcal{R}_{\varepsilon}^{(0)}$ everything is o.k. As for $\mathcal{R}_{\varepsilon}^{(1)}$, from the proof of Proposition 4.2 we see that it is enough to show that

$$
\exp \left(\frac{-\varepsilon}{2 d_{\alpha} \ell^{2}} \psi\left(x_{\alpha} \otimes y_{\alpha}\right) \cdot \operatorname{ad}_{\{,\}}\left(x_{\alpha} \otimes y_{\alpha}\right)\right)(x \otimes y)
$$

(for any $x, y$ in $U_{\varepsilon}$ ) is a rational section of the bundle $\mathcal{S}_{\varepsilon}^{(2 \ell)}$ on a $2 \ell$-fold ramified covering $\mathcal{H}^{(2 \ell)}$ of $H$ : this again amounts to perform some computations. In particular (cf. (4.4-7)) we are reduced to check the same for functions

$$
\begin{aligned}
& \exp \left(\frac{\log \left(1-{ }_{1} x_{\alpha} \cdot{ }_{2} y_{\alpha}\right)}{{ }_{1} x_{\alpha}} \cdot{ }_{1} \mathrm{e}_{\alpha}\right)\left({ }_{1} x \cdot{ }_{2} y\right) \\
& \exp \left(\frac{\log \left(1-{ }_{1} x_{\alpha} \cdot{ }_{2} y_{\alpha}\right)}{{ }_{2} y_{\alpha}} \cdot{ }_{2} \mathrm{f}_{\alpha}\right)\left({ }_{1} x \cdot{ }_{2} y\right)
\end{aligned}
$$

We deal with the first function above, the proof for the second following by symmetry. Since $\frac{\log \left(1-1_{1} x_{\alpha} \cdot 2 y_{\alpha}\right)}{1 x_{\alpha}} \cdot{ }_{1} \mathrm{e}_{\alpha}$ is a derivation of $\widehat{U_{\varepsilon}{ }^{\otimes 2}}$, its exponential is an automorphism of $\widehat{U_{\varepsilon}{ }^{\otimes 2}}$; now $\frac{\log \left(1-1 x_{\alpha} \cdot 2 y_{\alpha}\right)}{1 x_{\alpha}} \cdot \mathrm{e}_{\alpha}(1 \otimes y)=0$, whence $\exp \left(\frac{\log \left(1-1_{1} x_{\alpha} \cdot 2 y_{\alpha}\right)}{1 x_{\alpha}} \cdot{ }_{1} \mathrm{e}_{\alpha}\right)(1 \otimes y)=1 \otimes y$, for all $y \in U_{\varepsilon}$; therefore we have only to compute $\exp \left(\frac{\log \left(1-{ }_{1} x_{\alpha} \cdot 2 y_{\alpha}\right)}{{ }_{1} x_{\alpha}} \cdot{ }_{1} \mathrm{e}_{\alpha}\right)(x \otimes 1)$ for 
$x={ }_{1} x \in U_{\varepsilon}$ : in particular, it is enough to take $x$ to be a generator of $U_{\varepsilon}$, namely $x \in\left\{F_{i}, L_{\lambda}, E_{j} \mid i, j=1, \ldots, n ; \lambda \in P\right\}$.

Like in the proof of [DK], Proposition 3.5, exploiting the braid group action we can restrict to the case of simple roots $\alpha=\alpha_{i}, i=1, \ldots, n$ (thus we set ${ }_{1} \bar{E}_{i}:={ }_{1} \bar{E}_{\alpha_{i}}$, ${ }_{1} \mathrm{e}_{i}:={ }_{1} \mathrm{e}_{\alpha_{i}}$, and so on), using formulas

$$
\begin{aligned}
& { }_{1} \bar{E}_{\alpha}=T_{w}\left({ }_{1} \bar{E}_{i}\right), \quad{ }_{1} \bar{F}_{\alpha}=T_{w}\left({ }_{1} \bar{F}_{i}\right) \quad \text { for } \quad \alpha=w\left(\alpha_{i}\right) \\
& { }_{1} \mathrm{e}_{\alpha}=T_{w} \circ{ }_{1} \mathrm{e}_{i} \circ T_{w}^{-1}, \quad{ }_{1} \mathrm{f}_{\alpha}=T_{w} \circ{ }_{1} \mathrm{f}_{i} \circ T_{w}^{-1} \quad \text { for } \alpha=w\left(\alpha_{i}\right)
\end{aligned}
$$

(cf. [DK], §3.4), where $T_{w}$ denotes the unique element of the braid group associated to $w \in W$. Moreover, from direct computation or resuming formulas in the proof of [DK], Proposition 3.5, we get, mutatis mutandis,

$$
\begin{aligned}
& \exp \left(t \cdot{ }_{1} \mathrm{e}_{i}\right)\left({ }_{1} L_{\lambda}\right)=e^{\left(\left\langle\alpha_{i} \mid \lambda\right\rangle / 2 \ell\right) \cdot t \cdot{ }_{1} x_{i}} \cdot{ }_{1} L_{\lambda} \\
& \exp \left(t \cdot{ }_{1} \mathrm{e}_{i}\right)\left({ }_{1} \bar{F}_{j}\right)= \\
& \quad={ }_{1} \bar{F}_{j}-\delta_{i j}\left(\frac{e^{-t \cdot{ }_{1} x_{i} / \ell}-1}{{ }_{1} x_{i}} \cdot \varepsilon^{d_{i}} L_{\alpha_{i}}+\frac{e^{t \cdot 1 x_{i} / \ell}-1}{{ }_{1} x_{i}} \cdot \varepsilon^{-d_{i}} L_{-\alpha_{i}}\right) \cdot{ }_{1} \bar{E}_{i}^{\ell-1}
\end{aligned}
$$

for any indeterminate $t$ which commutes with ${ }_{1} \bar{E}_{i}$ (where $\left\langle\alpha_{i} \mid \lambda\right\rangle:=2\left(\alpha_{i} \mid \lambda\right) /\left(\alpha_{i} \mid \alpha_{i}\right) \in$ $\mathbb{Z}$ ); when instead of $t$ we have the meromomorphic function $\frac{\log \left(1-x_{1} x_{i} \cdot y_{i}\right)}{1 x_{i}}$ (which does commute with ${ }_{1} \bar{E}_{i}$ !) the previous formulas give

$$
\begin{aligned}
& \exp \left(\frac{\log \left(1-{ }_{1} x_{i} \cdot{ }_{2} y_{i}\right)}{{ }_{1} x_{i}} \cdot{ }_{1} \mathrm{e}_{i}\right)\left({ }_{1} L_{\lambda}\right)=\left(1-{ }_{1} x_{i} \cdot{ }_{2} y_{i}\right)^{\frac{\left\langle\alpha_{i} \mid \lambda\right\rangle}{2 \ell}} \\
& \exp \left(\frac{\log \left(1-{ }_{1} x_{i} \cdot{ }_{2} y_{i}\right)}{{ }_{1} x_{i}} \cdot{ }_{1} \mathrm{e}_{i}\right)\left({ }_{1} \bar{F}_{j}\right)={ }_{1} \bar{F}_{j}- \\
& \quad-\delta_{i j}\left(\frac{\left(1-{ }_{1} x_{i} \cdot{ }_{2} y_{i}\right)^{-1 / \ell}-1}{{ }_{1} x_{i}} \cdot \varepsilon^{d_{i}} L_{\alpha_{i}}+\frac{\left(1-{ }_{1} x_{i} \cdot{ }_{2} y_{i}\right)^{1 / \ell}-1}{{ }_{1} x_{i}} \cdot \varepsilon^{-d_{i}} L_{-\alpha_{i}}\right) \cdot{ }_{1} \bar{E}_{i}^{\ell-1}
\end{aligned}
$$

and both these are rational functions on $\mathcal{S}_{\varepsilon}^{(2 \ell)} \times \mathcal{S}_{\varepsilon}^{(2 \ell)}$.

Now we are left with the case $x=\bar{E}_{j}, j=1, \ldots, n$. Consider ${ }_{1} \mathrm{e}_{i}\left({ }_{1} \bar{E}_{j}\right)$; if $a_{i j}=2$ (i. e. $i=j$ ) or $a_{i j}=0$ we have ${ }_{1} \mathrm{e}_{i}\left({ }_{1} \bar{E}_{j}\right)=0$, hence

$$
\exp \left(\frac{\log \left(1-{ }_{1} x_{i} \cdot{ }_{2} y_{i}\right)}{{ }_{1} x_{i}} \cdot{ }_{1} \mathrm{e}_{i}\right)\left({ }_{1} \bar{E}_{j}\right)={ }_{1} \bar{E}_{j} .
$$

Therefore we are reduced to make computations in the connected rank 2 case. To this end, we will follow conventions and notations of [DP], Appendix, and skip for a while left bottom indices "1" (i. e. ${ }_{1} \bar{E}_{i}=\bar{E}_{i}$, etc.).

We develop the $A_{2}$ case; the procedure is the same in the remaining cases but the computations are longer (cf. also the Remark after the proof).

In this case we have $d_{1}=1=d_{2}$. Define the root vector $E_{12}:=E_{\alpha_{1}+\alpha_{2}} \in \mathcal{U}_{q}^{P}(\mathfrak{g})$ as

$$
E_{\alpha_{12}} \equiv E_{12}:=T_{1}\left(E_{2}\right)=-E_{1} E_{2}+q^{-1} E_{2} E_{1}
$$


then we have

$$
E_{2} E_{1}=q E_{1} E_{2}+q E_{12}, \quad E_{12} E_{1}=q^{-1} E_{1} E_{12}
$$

Let $\mathbb{C}(q)\left(E_{1}\right)$ be the field of rational functions in the indeterminate $E_{1}$ with coefficients in $\mathbb{C}(q)$; let $M$ be the $\mathbb{C}(q)\left(E_{1}\right)$-vector space with basis $\left\{\bar{E}_{2}, \bar{E}_{12}\right\}$ : then (4.12) tells us that the operation $\rho_{E_{1}}$ of right multiplication by $E_{1}$ yields an endomorphism of $M$ defined by the matrix (with respect to the ordered $\mathbb{C}(q)\left(E_{1}\right)$-basis $\left\{\bar{E}_{2}, \bar{E}_{12}\right\}$ )

$$
\left(\begin{array}{cc}
q E_{1} & 0 \\
q & q^{-1} E_{1}
\end{array}\right)
$$

therefore multiplication by $E_{1}^{n}$ yields the endomorphism of $M$ defined by the matrix

$$
\left(\begin{array}{cc}
q E_{1} & 0 \\
q & q^{-1} E_{1}
\end{array}\right)^{n}=\left(\begin{array}{cc}
\left(q E_{1}\right)^{n} & 0 \\
q[n]_{q} \cdot E_{1}^{n-1} & \left(q^{-1} E_{1}\right)^{n}
\end{array}\right) .
$$

Thus for $\mathrm{e}_{1}\left(\bar{E}_{2}\right)$ we have

$$
\begin{gathered}
\mathrm{e}_{1}\left(\bar{E}_{2}\right):=\left.\left[E_{1}^{(\ell)}, \bar{E}_{2}\right]\right|_{q=\varepsilon}=\left.\frac{E_{1}^{\ell} \bar{E}_{2}-\bar{E}_{2} E_{1}^{\ell}}{[\ell]_{q} !}\right|_{q=\varepsilon}=\left.\frac{E_{1}^{\ell} \bar{E}_{2}-q^{\ell} E_{1}^{\ell} \bar{E}_{2}-q[\ell]_{q} E_{1}^{\ell-1} \bar{E}_{12}}{[\ell]_{q} !}\right|_{q=\varepsilon}= \\
=\left.\left(\frac{1-q^{\ell}}{q^{\ell}-q^{-\ell}} \cdot \frac{q-q^{-1}}{[\ell-1]_{q} !} \cdot E_{1}^{\ell} \bar{E}_{2}\right)\right|_{q=\varepsilon}-\left.\left(\frac{q}{[\ell-1]_{q} !} \cdot E_{1}^{\ell-1} \bar{E}_{12}\right)\right|_{q=\varepsilon}= \\
=-\frac{\bar{E}_{1}^{\ell}}{2 \ell} \cdot \bar{E}_{2}-\frac{\varepsilon}{\ell} \bar{E}_{1}^{\ell-1} \cdot \bar{E}_{12}=-\frac{x_{1}}{2 \ell} \cdot \bar{E}_{2}-\frac{\varepsilon}{\ell} \bar{E}_{1}^{\ell-1} \cdot \bar{E}_{12}
\end{gathered}
$$

(because $\left.\left.\frac{1}{[\ell-1]_{q} !}\right|_{q=\varepsilon}=\left.\frac{\left(q-q^{-1}\right)^{\ell-1}}{\prod_{s=0}^{\ell-1}\left(q^{s}-q^{-s}\right)}\right|_{q=\varepsilon}=\frac{\left(\varepsilon-\varepsilon^{-1}\right)^{\ell-1}}{\ell}\right)$; on the other hand, for $\mathrm{e}_{1}\left(\bar{E}_{12}\right)$, (4.12) gives

$$
\begin{aligned}
\mathrm{e}_{1}\left(\bar{E}_{12}\right):=\left.\left[E_{1}^{(\ell)}, \bar{E}_{12}\right]\right|_{q=\varepsilon}=\left.\frac{E_{1}^{\ell} \bar{E}_{12}-\bar{E}_{12} E_{1}^{\ell}}{[\ell]_{q} !}\right|_{q=\varepsilon}=\left.\frac{E_{1}^{\ell} \bar{E}_{2}-q^{-\ell} E_{1}^{\ell} \bar{E}_{12}}{[\ell]_{q} !}\right|_{q=\varepsilon}= \\
=\left.\left(\frac{1-q^{-\ell}}{q^{\ell}-q^{-\ell}} \cdot \frac{q-q^{-1}}{[\ell-1]_{q} !} \cdot E_{1}^{\ell} \bar{E}_{12}\right)\right|_{q=\varepsilon}=\frac{\bar{E}_{1}^{\ell}}{2 \ell} \cdot \bar{E}_{12}=\frac{x_{1}}{2 \ell} \cdot \bar{E}_{12}
\end{aligned}
$$

therefore we conclude that $\mathrm{e}_{1}$ restricts to an endomorphism of $M$ defined by the matrix

$$
\left(\begin{array}{cc}
-\frac{\bar{E}_{1}^{\ell}}{2 \ell} & 0 \\
-\frac{\varepsilon}{\ell} \cdot \bar{E}_{1}^{\ell-1} & \frac{\bar{E}_{1}^{\ell}}{2 \ell}
\end{array}\right)=\left(\begin{array}{cc}
-\frac{x_{1}}{2 \ell} & 0 \\
-\frac{\varepsilon}{\ell} \cdot \bar{E}_{1}^{\ell-1} & \frac{x_{1}}{2 \ell}
\end{array}\right)
$$

hence $\left.\mathrm{e}_{1}^{n}\right|_{M}=\left(\left.\mathrm{e}_{1}\right|_{M}\right)^{n}$ is given by the matrix

$$
\left(\begin{array}{cc}
-\frac{x_{1}}{2 \ell} & 0 \\
-\frac{\varepsilon}{\ell} \cdot \bar{E}_{1}^{\ell-1} & \frac{x_{1}}{2 \ell}
\end{array}\right)^{n}=\left(\begin{array}{cc}
\left(-\frac{x_{1}}{2 \ell}\right)^{n} & 0 \\
-\delta_{n \in(2 \mathbb{N}+1)} \cdot \frac{2 \varepsilon}{x_{1}} \cdot\left(\frac{x_{1}}{2 \ell}\right)^{n} \cdot \bar{E}_{1}^{\ell-1} & \left(\frac{x_{1}}{2 \ell}\right)^{n}
\end{array}\right)
$$


(for all $n \in \mathbb{N}$, where $\delta_{x \in Y}:=1$ for $x \in Y$ and $\delta_{x \in Y}:=0$ for $x \notin Y$ ), so that

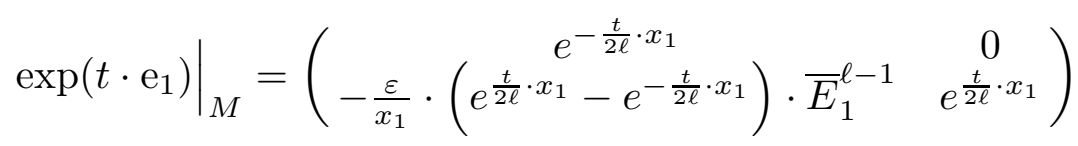

where $t$ denotes any indeterminate which commute with $E_{1}$; in particular for $t=\frac{\log \left(1-w_{1}\right)}{\bar{E}_{1}^{\ell}}$, with $w_{1}:={ }_{1} \bar{E}_{1}^{\ell} \cdot{ }_{2} \bar{F}_{1}^{\ell}={ }_{1} x_{1} \cdot{ }_{2} y_{1}$, we get

$$
\left.\exp \left(\frac{\log \left(1-w_{1}\right)}{\bar{E}_{1}^{\ell}} \cdot \mathrm{e}_{1}\right)\right|_{M}=\left(\begin{array}{cc}
\left(1-w_{1}\right)^{-\frac{1}{2 \ell}} & 0 \\
-\frac{\varepsilon}{\bar{E}_{1}^{\ell}} \cdot\left(\left(1-w_{1}\right)^{\frac{1}{2 \ell}}-\left(1-w_{1}\right)^{-\frac{1}{2 \ell}}\right) \cdot \bar{E}_{1}^{\ell-1} & \left(1-w_{1}\right)^{\frac{1}{2 \ell}}
\end{array}\right)
$$

thus

$$
\begin{gathered}
\exp \left(\frac{\log \left(1-{ }_{1} x_{1} \cdot{ }_{2} y_{1}\right)}{{ }_{1} x_{1}} \cdot{ }_{1} \mathrm{e}_{1}\right)\left({ }_{1} \bar{E}_{2}\right)= \\
=\left(1-{ }_{1} x_{1} \cdot{ }_{2} y_{1}\right)^{-\frac{1}{2 \ell}} \cdot{ }_{1} \bar{E}_{2}-\frac{\varepsilon}{{ }_{1} x_{1}} \cdot\left(\left(1-{ }_{1} x_{1} \cdot{ }_{2} y_{1}\right)^{\frac{1}{2 \ell}}-\left(1-{ }_{1} x_{1} \cdot{ }_{2} y_{1}\right)^{-\frac{1}{2 \ell}}\right) \cdot{ }_{1} \bar{E}_{1}{ }^{\ell-1} \cdot{ }_{1} \bar{E}_{12}
\end{gathered}
$$

which is a rational section of a $\mathcal{S}_{\varepsilon}^{(2 \ell)} \times \mathcal{S}_{\varepsilon}^{(2 \ell)}$, q. e. d.

As for $\exp \left(\frac{\log \left(1-x_{1} x_{2} \cdot y_{2}\right)}{1 x_{2}} \cdot{ }_{1} \mathrm{e}_{2}\right)$, everything comes from above by symmetry, namely because $\alpha_{2}=s_{1} s_{2}\left(\alpha_{1}\right)$ implies ${ }_{1} \bar{E}_{2}=T_{1} T_{2}\left({ }_{1} \bar{E}_{1}\right),{ }_{1} \bar{F}_{2}=T_{1} T_{2}\left({ }_{1} \bar{F}_{1}\right)$, and ${ }_{1} \mathrm{e}_{2}=$ $\left(T_{1} T_{2}\right) \circ{ }_{1} \mathrm{e}_{1} \circ\left(T_{1} T_{2}\right)^{-1}$; on the other hand, in the other cases of rank 2 (that is $B_{2}$ and $G_{2}$ ) such a symmetric situation does not occur, hence we must perform direct computation for $\exp \left(\frac{\log \left(1-{ }_{1} x_{2} \cdot y_{2}\right)}{{ }_{1} x_{2}} \cdot{ }_{1} \mathrm{e}_{2}\right)$ too (this is entirely similar, although longer, to the previous one).

Finally, it is clear that restricting to subalgebras $Z_{\varepsilon}$ and $Z_{0}$ we get (bi)rational Poisson automorphisms of their spectra, by the same argument of the end of the proof of Proposition 4.2.

Remark: the very (theoretical) reason why computations do work in all rank two cases, so that Theorem 4.3 does hold, lies in the availability of the commutation formulas for quantum root vectors (the so-called Levendorskij-Soibel'man formulas, cf. [DP], Theorem $9.3)$, strictly related with the existence of a convex ordering on the set of positive roots.

The previous result can be still improved when considering the central Hopf subalgebra $Z_{0}$, hence the Poisson group $H$, as the following shows:

Theorem 4.4. The birational Poisson automorphism $\mathcal{R}_{\varepsilon, \ell}^{*}: \mathcal{H}^{(2 \ell)} \times \mathcal{H}^{(2 \ell)} \rightarrow \mathcal{H}^{(2 \ell)} \times \mathcal{H}^{(2 \ell)}$ pushes down to a birational Poisson automorphism $\mathcal{R}_{\varepsilon, \ell}^{*}: \mathcal{H}^{(2)} \times \mathcal{H}^{(2)} \rightarrow \mathcal{H}^{(2)} \times \mathcal{H}^{(2)}$, independent of $\ell$, of a 2-fold ramified covering $\mathcal{H}^{(2)} \times \mathcal{H}^{(2)}$ of $H \times H ;$ moreover, $\mathcal{R}_{\varepsilon, \ell}^{*} \neq \tau^{(2)}$ (the "twist" map of $\mathcal{H}^{(2)} \times \mathcal{H}^{(2)}$ ), and $\mathcal{R}_{\varepsilon, \ell}^{*}$ enjoys the dual properties of (1.4-6): in particular, $m\left(\mathcal{R}_{\varepsilon, \ell}^{*}(x, y)\right)=y \cdot x$ for all $x, y \in \mathcal{H}^{(2)}$ (where $m$ and ". denote the product of $\mathcal{H}^{(2)}$ ), and a braid group action exists on $\times$-powers of $\mathcal{H}^{(2)}$. 
Proof. As for Theorem 4.3, the proof amounts to check that some series do converge on an appropriate covering. Namely, we have to check that

$$
\left.\exp \left(\frac{-\varepsilon}{2 d_{\alpha} \ell^{2}} \psi\left(x_{\alpha} \otimes y_{\alpha}\right) \cdot \operatorname{ad}_{\{,}\right\}\left(x_{\alpha} \otimes y_{\alpha}\right)\right)\left({ }_{1} w \otimes{ }_{2} w\right)
$$

does converge to a rational function on a covering $\mathcal{H}^{(2)} \times \mathcal{H}^{(2)}$ as claimed for all $\alpha \in R^{+}$ and for all ${ }_{i} w \in\left\{1,{ }_{i} x_{\beta},{ }_{i} z_{\lambda},{ }_{i} y_{\gamma} \mid \beta, \gamma \in R^{+} ; \lambda \in P\right\}, i=1,2$. This again amounts to prove the same for functions

$$
\begin{aligned}
& \exp \left(\frac{\log \left(1-{ }_{1} x_{\alpha} \cdot{ }_{2} y_{\alpha}\right)}{{ }_{1} x_{\alpha}} \cdot{ }_{1} \mathrm{e}_{\alpha}\right)\left({ }_{1} w\right) \\
& \exp \left(\frac{\log \left(1-{ }_{1} x_{\alpha} \cdot{ }_{2} y_{\alpha}\right)}{{ }_{2} y_{\alpha}} \cdot{ }_{2} \mathrm{f}_{\alpha}\right)\left({ }_{2} w\right)
\end{aligned}
$$

for all $\alpha$ and ${ }_{i} w$ like above. As for Theorem 4.3, we deal with the first function, the proof for the second one following by symmetry.

By the braid group action we can again reduce to the case of simple roots $\alpha=\alpha_{i}$. Furthermore (cf. [DK], §3.4, and [DP], §19), with respect to coordinates $x_{\gamma}:=\bar{E}_{\gamma}^{\ell}, z_{\lambda}:=$ $L_{\lambda}^{\ell}, y_{\gamma}:=\bar{F}_{\gamma}^{\ell}$, the formulas for derivations $\mathrm{e}_{\alpha}$ are independent of $\ell$ : therefore we can fix $\ell=1$ and perform computations in $U_{1}$.

Again direct computation (or formulas in the proof of [DK], Proposition 3.5) gives

$$
\exp \left(t \cdot{ }_{1} \mathrm{e}_{i}\right)\left({ }_{1} z_{\lambda}\right)=e^{\left(\left\langle\alpha_{i} \mid \lambda\right\rangle / 2\right) \cdot t \cdot{ }_{1} x_{i}} \cdot{ }_{1} z_{\lambda}
$$

for any indeterminate $t$ which commutes with ${ }_{1} \bar{E}_{i}={ }_{1} x_{i}$; then for $t=\frac{\log \left(1-{ }_{1} x_{i} \cdot{ }_{2} y_{i}\right)}{1 x_{i}}$ we have

$$
\exp \left(\frac{\log \left(1-{ }_{1} x_{i} \cdot{ }_{2} y_{i}\right)}{{ }_{1} x_{i}} \cdot{ }_{1} \mathrm{e}_{i}\right)\left({ }_{1} z_{\lambda}\right)=\left(1-{ }_{1} x_{i} \cdot{ }_{2} y_{i}\right)^{\frac{\left\langle\alpha_{i} \mid \lambda\right\rangle}{2}} \cdot{ }_{1} z_{\lambda}
$$

which is a rational function on a 2-fold ramified covering $\mathcal{H}^{(2)} \times \mathcal{H}^{(2)}$ of $H \times H$.

Now consider $\exp \left(\frac{\log \left(1-{ }_{1} x_{i} \cdot{ }_{2} y_{i}\right)}{{ }_{1} x_{i}} \cdot{ }_{1} \mathrm{e}_{i}\right)\left({ }_{1} x_{\gamma}\right)$, with $\gamma \in R^{+}$(notice that now simple root vectors $\bar{E}_{j}=x_{j}(j=1, \ldots, n)$ are not enough to generate $U_{1}^{+}$(the "positive part" of $\left.U_{1}\right)$ : we do need all root vectors $\left.\bar{E}_{\gamma}=x_{\gamma}, \gamma \in R^{+}\right)$. For any fixed pair $(\alpha, \gamma)$ of positive roots, let us denote by $R_{\beta, \gamma}^{+}$the rank 2 root system spanned by $\{\alpha, \gamma\}$ in $R^{+}$. The following is well known (cf. e. g. [DP], first Lemma of $§ 15.4$ ):

Claim: For any fixed pair $\left(\alpha_{i}, \gamma\right)$ of positive roots with $\alpha_{i}$ simple, there exists $w \in W$ and $\alpha_{1}, \alpha_{2} \in R^{+}$such that $w\left(R_{\alpha_{1}, \alpha_{2}}^{+}\right)=R_{\alpha_{i}, \gamma}^{+}$and $w\left(\alpha_{1}\right)=\alpha_{i}$.

Thanks to Claim and (4.10) we are reduced to make computations in the rank 2 case; the same holds when considering $\exp \left(\frac{\log \left(1-{ }_{1} x_{i} \cdot 2 y_{i}\right)}{1 x_{i}} \cdot{ }_{1} \mathrm{e}_{i}\right)\left({ }_{1} y_{\gamma}\right)$, with $\gamma \in R^{+}$(now again negative simple root vectors $\bar{F}_{j}=y_{j}(j=1, \ldots, n)$ are not enough to generate $U_{1}^{-}$(the "negative part" of $U_{1}$ ): we do need all negative root vectors $\bar{F}_{\gamma}=y_{\gamma}, \gamma \in R^{+}$). We denote by $T$ the type of a root system of rank 2 (hence $T \in\left\{A_{1} \times A_{1}, A_{2}, B_{2}, G_{2}\right\}$ ). 
$T=A_{1} \times A_{1}:$ First of all, since $\mathrm{e}_{j}\left(x_{j}\right)=\mathrm{e}_{j}\left(\bar{E}_{j}\right)=0(j=1,2)$, we have

$$
\exp \left(\frac{\log \left(1-{ }_{1} x_{j} \cdot{ }_{2} y_{j}\right)}{{ }_{1} x_{j}} \cdot{ }_{1} \mathrm{e}_{j}\right)\left({ }_{1} x_{j}\right)={ }_{1} x_{j} \quad(j=1,2) ;
$$

second, since $a_{12}=0$, we have $\mathrm{e}_{i}\left(x_{j}\right)=0$ (for $i, j \in\{1,2\}, i \neq j$ ) whence

$$
\exp \left(\frac{\log \left(1-{ }_{1} x_{i} \cdot{ }_{2} y_{i}\right)}{{ }_{1} x_{i}} \cdot{ }_{1} \mathrm{e}_{i}\right)\left({ }_{1} x_{j}\right)={ }_{1} x_{j}
$$

(for $i, j \in\{1,2\}, i \neq j$ ) thus we are done with generators $x_{\alpha}$ 's.

As for negative root vectors $y_{\alpha}=\bar{F}_{\alpha}$, we have $\mathrm{e}_{i}\left(y_{j}\right)=\delta_{i j} \cdot\left(z_{\alpha_{i}}-z_{-\alpha_{i}}\right)$, whence

$$
\mathrm{e}_{i}^{n}\left(y_{j}\right)=\delta_{i j} \cdot\left(\mathrm{e}_{i}^{n-1}\left(z_{\alpha_{i}}\right)-\mathrm{e}_{i}^{n-1}\left(z_{-\alpha_{i}}\right)\right)=\delta_{i j} \cdot\left(\left(-x_{i}\right)^{n-1} \cdot z_{\alpha_{i}}-x_{i}^{n-1} \cdot z_{-\alpha_{i}}\right)
$$

(thanks to (4.14)) for all $n \in \mathbb{N}_{+}$, thus

$$
\exp \left(t \cdot{ }_{1} \mathrm{e}_{i}\right)\left({ }_{1} y_{j}\right)={ }_{1} y_{j}-\delta_{i j} \cdot\left(\frac{e^{-t \cdot{ }_{1} x_{i}}-1}{{ }_{1} x_{i}} \cdot z_{\alpha_{i}}+\frac{e^{t \cdot{ }_{1} x_{i}}-1}{{ }_{1} x_{i}} \cdot z_{-\alpha_{i}}\right)
$$

for any indeterminate $t$ which commutes with ${ }_{1} x_{i}$; for $t=\frac{\log \left(1-1 x_{i} \cdot 2 y_{i}\right)}{1 x_{i}}$ we get

$$
\begin{gathered}
\exp \left(\frac{\log \left(1-{ }_{1} x_{i} \cdot{ }_{2} y_{i}\right)}{{ }_{1} x_{i}} \cdot{ }_{1} \mathrm{e}_{i}\right)\left({ }_{1} y_{j}\right)= \\
={ }_{1} y_{j}-\delta_{i j} \cdot\left(\frac{\left(1-{ }_{1} x_{i} \cdot{ }_{2} y_{i}\right)^{-1}-1}{{ }_{1} x_{i}} \cdot z_{\alpha_{i}}+\frac{\left(1-{ }_{1} x_{i} \cdot{ }_{2} y_{i}\right)-1}{{ }_{1} x_{i}} \cdot z_{-\alpha_{i}}\right)
\end{gathered}
$$

which is a rational function on a 2-fold ramified covering $\mathcal{H}^{(2)} \times \mathcal{H}^{(2)}$ of $H \times H(=$ $\left.\operatorname{Spec}\left(Z_{0}\right) \times \operatorname{Spec}\left(Z_{0}\right)\right)$. Since for $T=A_{1} \times A_{1}$ we have $R^{+}=\left\{\alpha_{1}, \alpha_{2}\right\}$, we are done.

$T=A_{2}$ : We follow again conventions and notations of [DP], Appendix. In the present case we have $d_{1}=1=d_{2}$, and $R^{+}=\left\{\alpha_{1}, \alpha_{12}:=\alpha_{1}+\alpha_{2}, \alpha_{2}\right\}$, and we define the root vector $E_{12}:=-E_{1} E_{2}+q^{-1} E_{2} E_{1}$ (cf. (4.11)). For $\gamma=\alpha_{1}$ we have as above

$$
\exp \left(\frac{\log \left(1-{ }_{1} x_{1} \cdot{ }_{2} y_{1}\right)}{{ }_{1} x_{1}} \cdot{ }_{1} \mathrm{e}_{1}\right)\left({ }_{1} x_{1}\right)={ }_{1} x_{1} .
$$

Then let $M$ be the $\mathbb{C}(q)\left(E_{1}\right)$-vector space with basis $\left\{x_{2}, x_{12}\right\}=\left\{\bar{E}_{2}, \bar{E}_{12}\right\}$ : then (4.12) tells us that the operation of right multiplication by $E_{1}$ yields an endomorphism of $M$ defined by the matrix (with respect to $\left\{\bar{E}_{2}, \bar{E}_{12}\right\}$ )

$$
\left(\begin{array}{cc}
q E_{1} & 0 \\
q & q^{-1} E_{1}
\end{array}\right)
$$


Thus for $\mathrm{e}_{1}\left(x_{2}\right)$ we have

$$
\begin{aligned}
& \mathrm{e}_{1}\left(x_{2}\right):=\left.\left[E_{1}, \bar{E}_{2}\right]\right|_{q=1}=\left.\left(E_{1} \bar{E}_{2}-\bar{E}_{2} E_{1}\right)\right|_{q=1}=\left.\left(E_{1} \bar{E}_{2}-q E_{1} \bar{E}_{2}-q \bar{E}_{12}\right)\right|_{q=1}= \\
& =\left.\left(\frac{1-q}{q-q^{-1}} \cdot \bar{E}_{1} \bar{E}_{2}\right)\right|_{q=1}-\left.\left(q \cdot \bar{E}_{12}\right)\right|_{q=1}=-\frac{\bar{E}_{1}}{2} \cdot \bar{E}_{2}-\bar{E}_{12}=-\frac{x_{1}}{2} \cdot x_{2}-x_{12} ;
\end{aligned}
$$

on the other hand, for $\mathrm{e}_{1}\left(x_{12}\right),(4.12)$ gives

$$
\begin{gathered}
\mathrm{e}_{1}\left(x_{12}\right):=\left.\left[E_{1}, \bar{E}_{12}\right]\right|_{q=1}=\left.\left(E_{1} \bar{E}_{12}-\bar{E}_{12} E_{1}\right)\right|_{q=1}=\left.\left(E_{1} \bar{E}_{2}-q^{-1} E_{1} \bar{E}_{12}\right)\right|_{q=1}= \\
=\left.\left(\frac{1-q^{-1}}{q-q^{-1}} \cdot E_{1} \bar{E}_{12}\right)\right|_{q=1}=\frac{\bar{E}_{1}}{2} \cdot \bar{E}_{12}=\frac{x_{1}}{2} \cdot x_{12}
\end{gathered}
$$

therefore we conclude that $\mathrm{e}_{1}$ restricts to an endomorphism of $M$ defined by the matrix

$$
\left(\begin{array}{cc}
-\frac{x_{1}}{2} & 0 \\
-1 & \frac{x_{1}}{2}
\end{array}\right)
$$

hence $\left.\mathrm{e}_{1}^{n}\right|_{M}=\left(\left.\mathrm{e}_{1}\right|_{M}\right)^{n}$ is given by the matrix

$$
\left(\begin{array}{cc}
-\frac{x_{1}}{2} & 0 \\
-1 & \frac{x_{1}}{2}
\end{array}\right)^{n}=\left(\begin{array}{cc}
\left(-\frac{x_{1}}{2}\right)^{n} & 0 \\
-\delta_{n \in(2 \mathbb{N}+1)} \cdot \frac{2}{x_{1}} \cdot\left(\frac{x_{1}}{2}\right)^{n} & \left(\frac{x_{1}}{2}\right)^{n}
\end{array}\right)
$$

(for all $n \in \mathbb{N}$, so that

$$
\left.\exp \left(t \cdot \mathrm{e}_{1}\right)\right|_{M}=\left(\begin{array}{cc}
e^{-\frac{t}{2} \cdot x_{1}} & 0 \\
-\frac{1}{x_{1}} \cdot\left(e^{\frac{t}{2} \cdot x_{1}}-e^{-\frac{t}{2} \cdot x_{1}}\right. & e^{\frac{t}{2} \cdot x_{1}}
\end{array}\right)
$$

for any $t$ which commutes with $E_{1}$, and for $t=\frac{\log \left(1-{ }_{1} x_{1} \cdot 2 y_{1}\right)}{x_{1}}$ we have

$$
\begin{gathered}
\left.\exp \left(\frac{\log \left(1-{ }_{1} x_{1} \cdot{ }_{2} y_{1}\right)}{x_{1}} \cdot{ }_{1} \mathrm{e}_{1}\right)\right|_{M}= \\
=\left(\begin{array}{cc}
\left(1-{ }_{1} x_{1} \cdot{ }_{2} y_{1}\right)^{-\frac{1}{2}} & 0 \\
-\frac{1}{1 x_{1}} \cdot\left(\left(1-{ }_{1} x_{1} \cdot{ }_{2} y_{1}\right)^{\frac{1}{2}}-\left(1-{ }_{1} x_{1} \cdot{ }_{2} y_{1}\right)^{-\frac{1}{2}}\right) & \left(1-{ }_{1} x_{1} \cdot{ }_{2} y_{1}\right)^{\frac{1}{2}}
\end{array}\right)
\end{gathered}
$$

or, in other words,

$$
\begin{gathered}
\exp \left(\frac{\log \left(1-{ }_{1} x_{1} \cdot{ }_{2} y_{1}\right)}{{ }_{1} x_{1}} \cdot{ }_{1} \mathrm{e}_{1}\right)\left({ }_{1} x_{2}\right)= \\
=\left(1-{ }_{1} x_{1} \cdot{ }_{2} y_{1}\right)^{-\frac{1}{2}} \cdot{ }_{1} x_{2}-\frac{1}{{ }_{1} x_{1}} \cdot\left(\left(1-{ }_{1} x_{1} \cdot{ }_{2} y_{1}\right)^{\frac{1}{2}}-\left(1-{ }_{1} x_{1} \cdot{ }_{2} y_{1}\right)^{-\frac{1}{2}}\right) \cdot{ }_{1} x_{12} \\
\exp \left(\frac{\log \left(1-{ }_{1} x_{1} \cdot{ }_{2} y_{1}\right)}{{ }_{1} x_{1}} \cdot{ }_{1} \mathrm{e}_{1}\right)\left({ }_{1} x_{12}\right)=\left(1-{ }_{1} x_{1} \cdot{ }_{2} y_{1}\right)^{\frac{1}{2}} \cdot{ }_{1} x_{12}
\end{gathered}
$$


and these are rational functions on a 2-fold ramified covering $\mathcal{H}^{(2)} \times \mathcal{H}^{(2)}$ of $H \times H$, q. e. d.

For negative root vectors $y_{\alpha}=\bar{F}_{\alpha}$ 's, define $F_{\alpha_{12}} \equiv F_{12}:=T_{1}\left(F_{2}\right)=-F_{2} F_{1}+q F_{1} F_{2}$; then we have again $\mathrm{e}_{1}\left(y_{j}\right)=\delta_{1 j} \cdot\left(z_{\alpha_{1}}-z_{-\alpha_{1}}\right)(j=1,2)$, whence

$$
\begin{gathered}
\exp \left(\frac{\log \left(1-{ }_{1} x_{1} \cdot{ }_{2} y_{1}\right)}{{ }_{1} x_{1}} \cdot{ }_{1} \mathrm{e}_{1}\right)\left({ }_{1} y_{j}\right)= \\
={ }_{1} y_{j}-\delta_{1 j} \cdot\left(\frac{\left(1-{ }_{1} x_{1} \cdot{ }_{2} y_{1}\right)^{-1}-1}{{ }_{1} x_{1}} \cdot z_{\alpha_{1}}+\frac{\left(1-{ }_{1} x_{1} \cdot{ }_{2} y_{1}\right)-1}{{ }_{1} x_{1}} \cdot z_{-\alpha_{1}}\right)
\end{gathered}
$$

$(j=1,2)$ which is a rational function on the proper covering; this takes care of $\gamma=\alpha_{1}$ and $\gamma=\alpha_{2}$. At last, for $\gamma=\alpha_{12}:=\alpha_{1}+\alpha_{2}$, we have

$$
\begin{gathered}
\mathrm{e}_{1}\left(y_{12}\right)=\left.\left[E_{1}, \bar{F}_{12}\right]\right|_{q=1}=\left.\left(-F_{2}\left[E_{1}, \bar{F}_{1}\right]+q\left[E_{1}, \bar{F}_{1}\right] F_{2}\right)\right|_{q=1}= \\
=\left.\left(-\bar{F}_{2}\left(L_{\alpha_{1}}-L_{-\alpha_{1}}\right)+q\left(L_{\alpha_{1}}-L_{-\alpha_{1}}\right) \bar{F}_{2}\right)\right|_{q=1}=\left.\left(\frac{q^{2}-1}{q-q^{-1}} \cdot \bar{F}_{2} L_{\alpha_{1}}\right)\right|_{q=1}=z_{\alpha_{1}} \cdot y_{2}
\end{gathered}
$$

now, since for all $n \in \mathbb{N}$ we have

$$
\mathrm{e}_{1}^{n}\left(z_{\alpha_{1}} \cdot y_{2}\right)=\mathrm{e}_{1}^{n}\left(z_{\alpha_{1}}\right) \cdot y_{2}=\left(-x_{1}\right)^{n} \cdot z_{\alpha_{1}} \cdot y_{2}
$$

we get, for all $n \in \mathbb{N}_{+}$

$$
\mathrm{e}_{1}^{n}\left(y_{12}\right)=\mathrm{e}_{1}^{n-1}\left(z_{\alpha_{1}} \cdot y_{2}\right)=\mathrm{e}_{1}^{n-1} \cdot z_{\alpha_{1}} y_{2}=\left(-x_{1}\right)^{n-1} \cdot z_{\alpha_{1}} y_{2}=-\frac{\left(-x_{1}\right)^{n}}{x_{1}} \cdot z_{\alpha_{1}} y_{2}
$$

whence $\exp \left(t \cdot \mathrm{e}_{1}\right)\left(y_{12}\right)=y_{12}-\frac{e^{-t \cdot x_{1}}-1}{x_{1}} \cdot z_{\alpha_{1}} y_{2}$ and finally

$$
\exp \left(\frac{\log \left(1-{ }_{1} x_{1} \cdot{ }_{2} y_{1}\right)}{{ }_{1} x_{1}} \cdot{ }_{1} \mathrm{e}_{1}\right)\left({ }_{1} y_{12}\right)={ }_{1} y_{12}-\frac{\left(1-{ }_{1} x_{1} \cdot{ }_{2} y_{1}\right)^{-1}-1}{{ }_{1} x_{1}} \cdot{ }_{1} z_{\alpha_{1}} \cdot{ }_{1} y_{2}
$$

the latter being a rational function on the covering $\mathcal{H}^{(2)} \times \mathcal{H}^{(2)}$ of $H \times H$, q. e. d.

As for $\exp \left(\frac{\log \left(1-{ }_{1} x_{2} \cdot 2\right.}{\left.x_{2} y_{2}\right)} \cdot{ }_{1} \mathrm{e}_{2}\right)$, everything follows by symmetry; on the other hand, in cases $B_{2}$ and $G_{2}$ such a symmetric situation does not occur, hence we must perform direct computation for $\exp \left(\frac{\log \left(1-{ }_{1} x_{2} \cdot{ }_{2} y_{2}\right)}{{ }_{1} x_{2}} \cdot{ }_{1} \mathrm{e}_{2}\right)$ too (which is completely similar, although quite longer, to the previous one).

We stress the fact that the proof of Theorem 4.4 above also contains the proof of the following one, which means that the adjoint action of the $R$-matrix does specialize for $q \rightarrow 1$ to something more than formal, with a very precise geometric meaning: 
Theorem 4.5. For the braided Hopf algebra $\left(F[H], \mathcal{R}_{1}\right)=\left(U_{1}, \mathcal{R}_{1}\right)$ the "formal automorphism" $\mathcal{R}_{1}$ is in fact an effective Poisson automorphism of the field of rational functions on $\mathcal{H}^{(2)} \times \mathcal{H}^{(2)}$. In other words, $\mathcal{R}_{1}$ defines a Poisson birational automorphism $\mathcal{R}_{1}^{*}$ of $\mathcal{H}^{(2)} \times \mathcal{H}^{(2)}$ which enjoys the dual properties of $(1.4-6)$, in particular $m\left(\mathcal{R}_{1}^{*}(x, y)\right)=y \cdot x$ for all $x, y \in \mathcal{H}^{(2)}$, and a braid group action exists on $\times$-powers of $\mathcal{H}^{(2)}$.

4.6. Recall that, by general theory, one has $\operatorname{Spec}(A \otimes B)=\operatorname{Spec}(A) \times \operatorname{Spec}(B)$ for all associative unital algebras $A$ and $B$; moreover, if $M$ and $N$ are Poisson manifolds then $M \times N$ is a Poisson manifold too, whose symplectic leaves are all the products of symplectic leaves of $M$ and $N$. In our context this implies that the symplectic leaves of $H^{\times 2}\left(\operatorname{resp} .\left(\mathcal{H}^{(N)}\right)^{\times 2}\right)$ are all the products of symplectic leaves of $H$ (resp. $\left.\mathcal{H}^{(N)}\right)$.

Let $N \in\{2 \ell, \infty\}$. Let us denote by $\mathcal{Z}_{0}$ the algebra of meromorphic functions on $\mathcal{H}^{(N)}$. As we said, we can look at $S_{\varepsilon}$ as a sheaf of algebras over $H$; similarly, we can look at $\mathcal{S}_{\varepsilon}^{(N)}$ (the $N$-fold covering of $S_{\varepsilon}$ ) as a sheaf of algebras over $\mathcal{H}^{(N)}$ : its algebra of meromorphic sections $\mathcal{U}_{\varepsilon}$ is then $\mathcal{U}_{\varepsilon}=\mathcal{Z}_{0} \otimes_{Z_{0}} U_{\varepsilon}$. Now represent the elements of $\mathcal{H}^{(N)}=\operatorname{Spec}\left(\mathcal{Z}_{0}\right)$ as maximal ideals of $\mathcal{Z}_{0}$, and let $\mathfrak{m} \in \mathcal{H}^{(N)}$ : the fibre over $\mathfrak{m}$ of our sheaf is then $\mathcal{U}_{\varepsilon} / \mathfrak{m} \mathcal{U}_{\varepsilon}$. Similarly, the fibre over $(\mathfrak{m}, \mathfrak{n}) \in \mathcal{H}^{(N)} \times \mathcal{H}^{(N)}$ (of the sheaf of algebras $\mathcal{S}_{\varepsilon}^{(N)} \times \mathcal{S}_{\varepsilon}^{(N)}$ over $\left.\mathcal{H}^{(N)} \times \mathcal{H}^{(N)}\right)$ is $\left(\mathcal{U}_{\varepsilon} \otimes \mathcal{U}_{\varepsilon}\right) /\left(\mathfrak{m} \mathcal{U}_{\varepsilon} \otimes \mathcal{U}_{\varepsilon}+\mathcal{U}_{\varepsilon} \otimes \mathfrak{n} \mathcal{U}_{\varepsilon}\right)$.

Proposition 4.7. $\mathcal{R}_{\varepsilon, N}^{*}$ is a meromorphic automorphism of $\mathcal{S}_{\varepsilon}^{(N)} \times \mathcal{S}_{\varepsilon}^{(N)}$ as a fibre bundle over $\mathcal{H}^{(N)} \times \mathcal{H}^{(N)}$ with respect to the meromorphic automorphism $\left.\mathcal{R}_{\varepsilon, N}^{*}\right|_{\mathcal{H}^{(N)} \times \mathcal{H}^{(N)}}$ of the base space $\mathcal{H}^{(N)} \times \mathcal{H}^{(N)}$; in other words, the following diagram is commutative

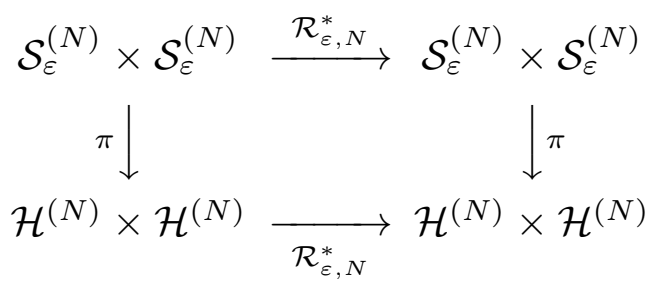

where $\pi: \mathcal{S}_{\varepsilon}^{(N)} \times \mathcal{S}_{\varepsilon}^{(N)} \longrightarrow \mathcal{H}^{(N)} \times \mathcal{H}^{(N)}$ is the projection map of the fibre bundle. In particular $\mathcal{R}_{\varepsilon, N}^{*}$ leaves invariant the fibres of $\mathcal{S}_{\varepsilon}^{(N)} \times \mathcal{S}_{\varepsilon}^{(N)}$ over symplectic leaves of $\mathcal{H}^{(N)} \times$ $\mathcal{H}^{(N)}$ (i. e. the preimages, with respect to $\pi$, of symplectic leaves of $\mathcal{H}^{(N)} \times \mathcal{H}^{(N)}$ ).

Proof. This is more or less trivial, by construction. Let $\mathfrak{s}=(\mathfrak{m}, \mathfrak{n}) \in \operatorname{Spec}\left(\mathcal{Z}_{0}{ }^{\otimes 2}\right)=$ $\mathcal{H}^{(N)} \times \mathcal{H}^{(N)}$ be a maximal ideal of $\mathcal{Z}_{0}{ }^{\otimes 2} ;$ then its fibre $\mathcal{U}_{\varepsilon}{ }^{\otimes 2} / \mathfrak{s} \mathcal{U}_{\varepsilon}{ }^{\otimes 2}$ is mapped by $\mathcal{R}_{\varepsilon, N}^{*}$ onto $\mathcal{R}_{\varepsilon, N}^{*}\left(\mathcal{U}_{\varepsilon}{ }^{\otimes 2} / \mathfrak{s} \mathcal{U}_{\varepsilon}{ }^{\otimes 2}\right)=\mathcal{U}_{\varepsilon}{ }^{\otimes 2} / \mathcal{R}_{\varepsilon}{ }^{-1}(\mathfrak{s}) \mathcal{U}_{\varepsilon}{ }^{\otimes 2}$, whence everything easily follows. 


\title{
REFERENCES
}

[DD] I. Damiani, C. De Concini, Quantum groups and Poisson groups, in W. Baldoni, M. Picardello (eds.), Representations of Lie groups and quantum groups, Longman Scientific \& Technical.

[DK] C. De Concini, V. G. Kac, Representations of Quantum Groups at Roots of 1, in Colloque Dixmier 1989, Progr. in Math. 92 (1990), 471 - 506.

[DKP] C. De Concini, V. G. Kac, C. Procesi, Quantum coadjoint action, Jour. Am. Math. Soc. 5 (1992), $151-189$.

[DL] C. De Concini, V. Lyubashenko, Quantum function algebra at roots of 1, Adv. in Math. 108 (1994), $205-262$.

[DP] C. De Concini, C. Procesi, Quantum Groups, in L. Boutet de Monvel, C. De Concini, C. Procesi, P. Schapira, M. Vergne (eds.), D-modules, Representation Theory, and Quantum Groups, Lectures Notes in Mathematics 1565, Springer \& Verlag, Berlin-Heidelberg-New York, 1993.

[Dr] V. G. Drinfeld, Quantum Groups, in Proc. Intern. Congress of Math. (Berkeley, 1986), AMS 1987, pp. $798-820$.

[Ex] H. Exton, q-Hypergeometric Functions and Applications, Ellis Hordwood Series Mathematics and its Applications, Ellis Hordwood Limited 1983.

[GR] G. Gasper, M. Rahman, Basic hypergeometric series, Encyclopedia of Mathematics and its Applications 35, Cambridge University Press, 1990.

[Ji] M. Jimbo, A q-difference analogue of $U(\mathfrak{g})$ and the Yang-Baxter equation, Lett. Math. Phys. 10 (1985), 63-69.

[KR] A. N. Kirillov, N. Reshetikin, q-Weyl Group and a Multiplicative Formula for Universal R-Matrices, Comm. Math. Phys. 134 (1990), 421-431.

[LS] S. Z. Levendorskij, Ya. S. Soibel'man, Some applications of the quantum Weyl groups, J. Geom. Physics 7 (1990), n ${ }^{\circ} 2,241-254$.

[Re] N. Reshetikin, Quasitriangularity of quantum groups at roots of 1, Commun. Math. Phys. 170 (1995), 79-99.

[Ta] T. Tanisaki, Killing forms, Harish-Chandra Isomorphisms, and Universal R-Matrices for Quantum Algebras, Internat. J. Modern Phys. A 7, Suppl. 1 B (1992), 941-961.

\author{
Dipartimento di Matematica, istituto "G. Castelnuovo" \\ Università DI ROMA "LA SAPIEnZA" \\ Piazzale Aldo Moro 5 \\ 00185 ROMA — ITALY \\ E-MAIL ADDRESS: GAVARINI@MAT.UNIROMA1.IT
}

